\title{
Tolerancing, alignment and test of the Transiting Exoplanet Survey Satellite (TESS) optical assembly
}

Brian Primeau, Michael Chrisp, Gregory Balonek, Christian Chesbrough, James Andre, Kristin Clark 


\section{Camera Structure Assembly (CSA)}

- Four wide field-of-view cameras with flexure mounts

- Camera Plate Assembly

- Camera Plate

- Bipods

- Purge Manifold

- Electrical and thermal harnesses

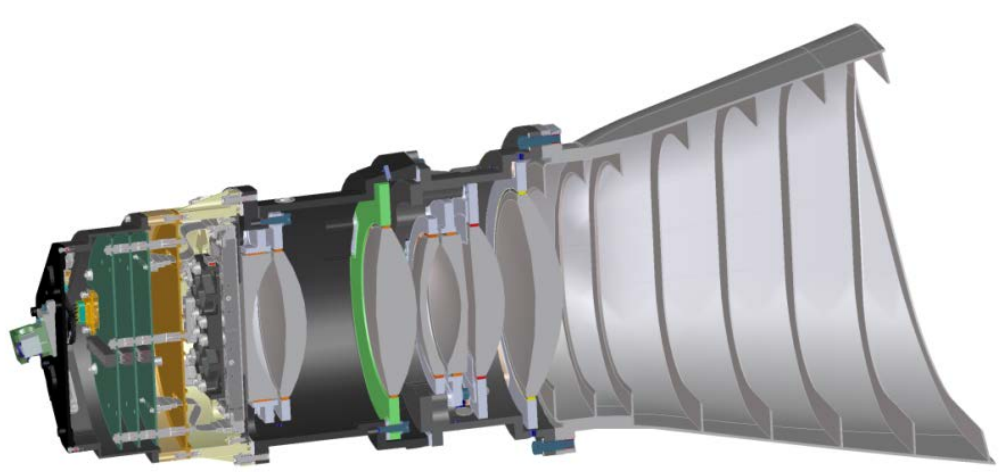

- Detector Assembly

- Dedicated Focal Plane Electronics

- CCD focal plane 4096x4096 pixels

- Lens Assembly

- $24^{\circ} \times 24^{\circ}$ FOV (>90\% sky coverage)

- $146 \mathrm{~mm}$ focal length $f / 1.4$

- Optimized over 600-1000 nm

- Lens Hoods $\left(12^{\circ}, 36^{\circ}\right)$

- Reduce scattered light

- Thermal radiator 
- Highly Elliptical Orbit provides extremely stable thermal environment

- Attitude change for data downlink creates a temperature pulse

- Wide field-of-view and step stare observing provide near full sky coverage

- Science orbit instrument pointing fixed in inertial space

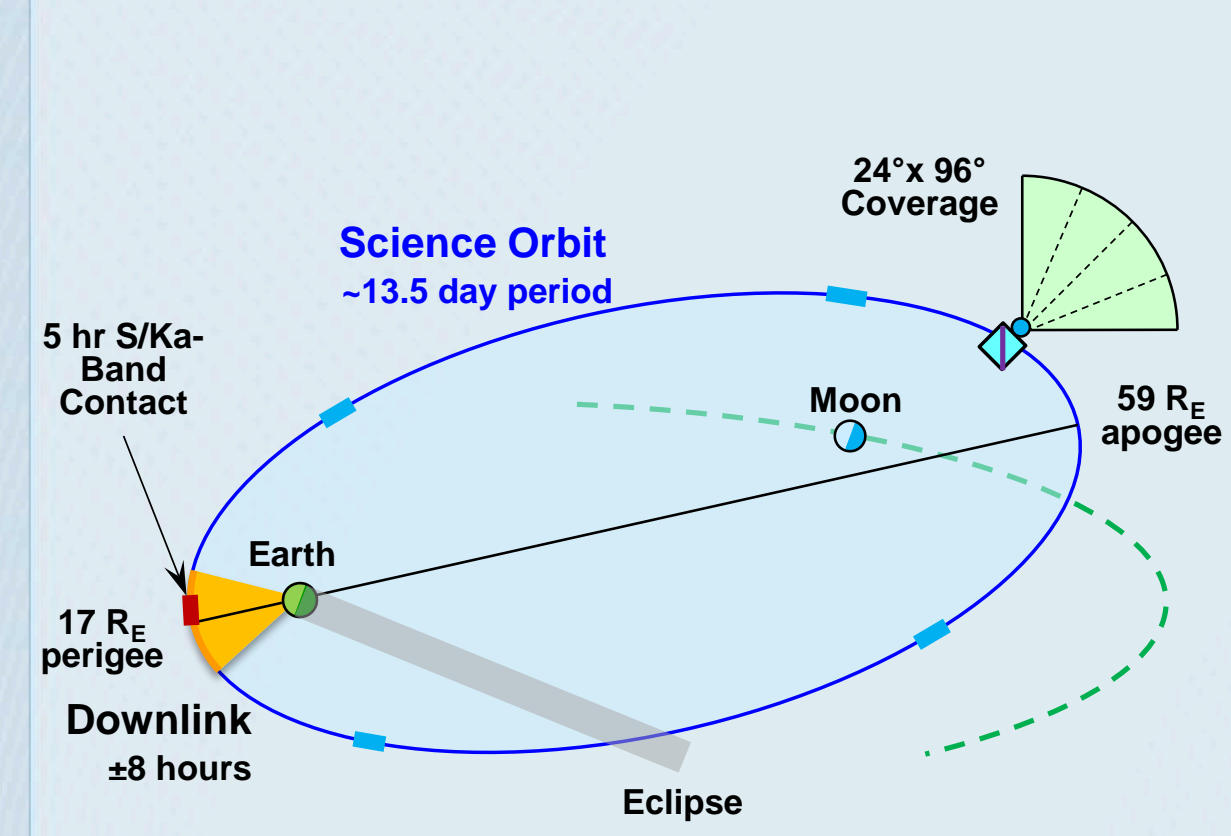

TESS Orbit

TESS 2-year sky coverage map

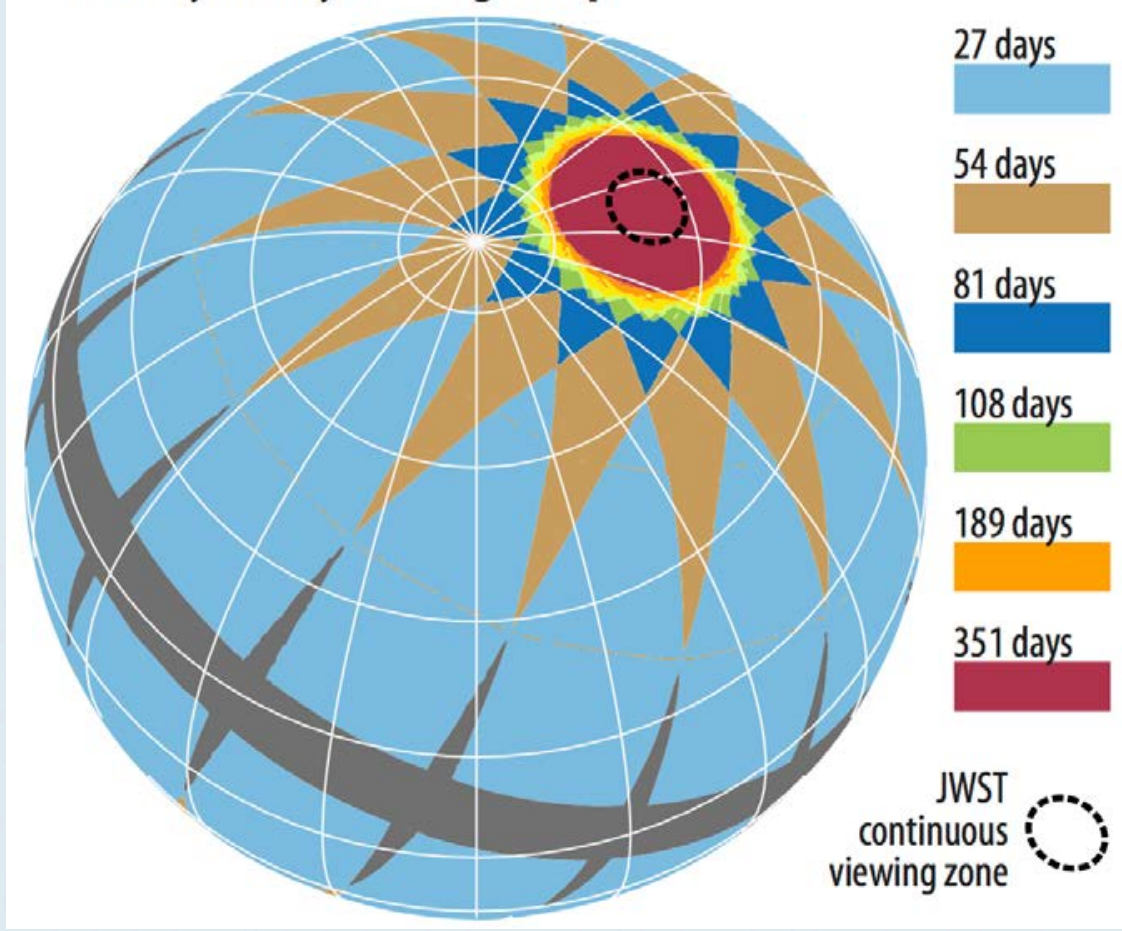




\section{Optical Design Overview}

- Lens Assembly

- $24^{\circ} \times 24^{\circ} \mathrm{FOV}$

- $146 \mathrm{~mm}, \mathrm{f} / 1.4$ lens

- $E P D=105 \mathrm{~mm}$

- 7 elements

- Two aspheric surfaces

- Optimized over 600-1000 nm
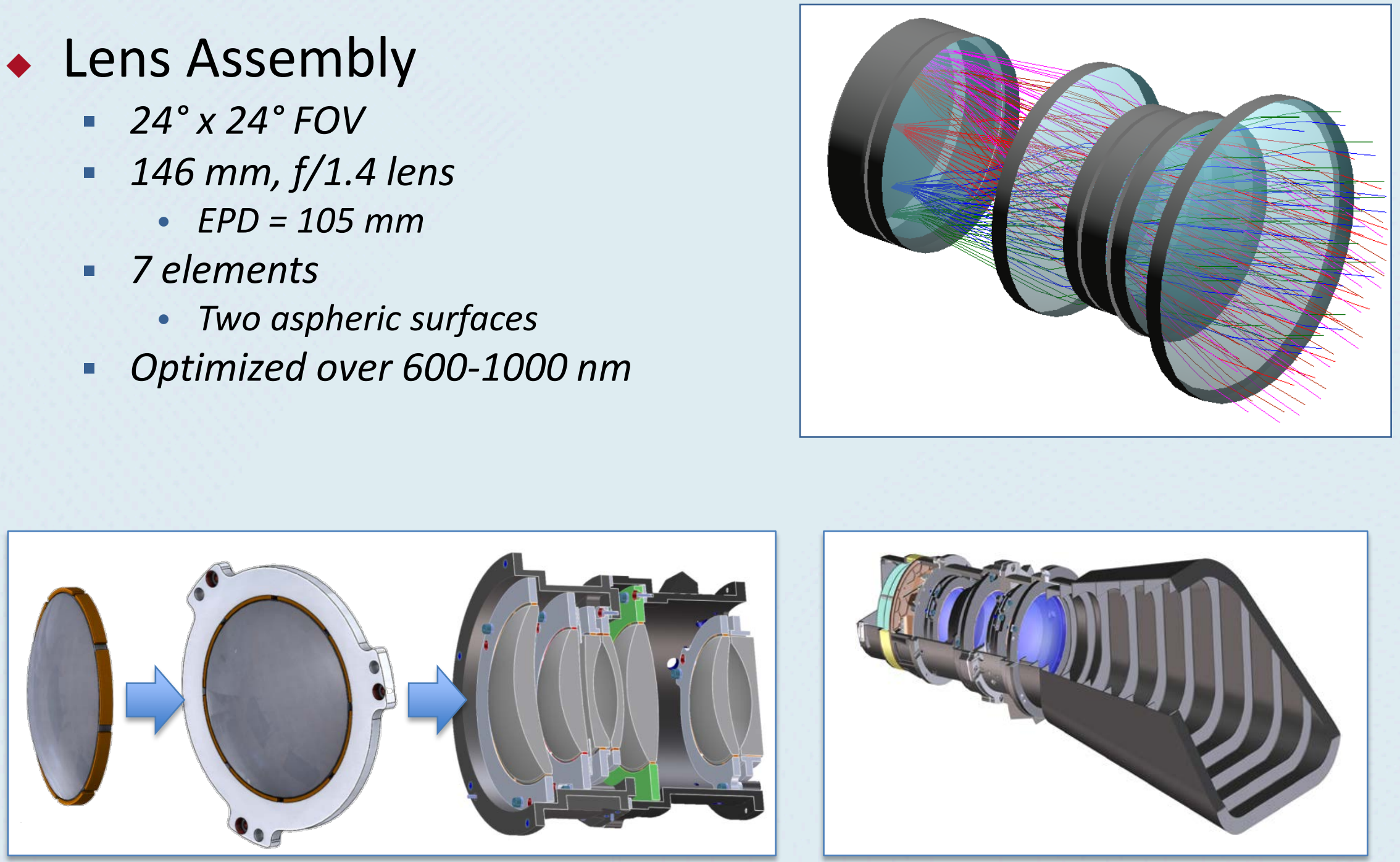


\section{Lens Assembly Design Tolerances}

\section{Specification / As-Built RRU}

\begin{tabular}{|c|c|c|c|c|c|c|c|c|c|c|}
\hline $\begin{array}{l}\text { L } \\
\text { e } \\
\text { n } \\
\text { s }\end{array}$ & Sur. & $\begin{array}{l}\text { Fringes } \\
\text { (power) }\end{array}$ & $\begin{array}{c}\text { Fringes } \\
\text { (irregularity) }\end{array}$ & $\begin{array}{c}\mathrm{dN} \\
\text { (melt comp.) }\end{array}$ & $\begin{array}{c}\mathrm{dV} \\
\text { (melt comp.) }\end{array}$ & $\begin{array}{c}\text { Lens } \\
\text { wedge } \\
\text { (ETD } \mu \mathrm{m} \text { ) }\end{array}$ & $\begin{array}{l}\text { Lens } \\
\text { thickness } \\
\text { ( } \mu \mathrm{m})\end{array}$ & $\begin{array}{c}\text { Axial } \\
\text { position } \\
\text { ( } \mu \mathrm{m})\end{array}$ & $\begin{array}{l}\text { Radial } \\
\text { decenter } \\
\text { ( } \mu \mathrm{m})\end{array}$ & $\begin{array}{l}\text { Lens tilt } \\
\text { (arc min) }\end{array}$ \\
\hline \multirow[t]{2}{*}{1} & 1 & $3 / 0.52$ & $0.5 / 0.28$ & \pm 0.00004 & $\pm 0.02 \%$ & $5 / 3$ & $\pm 25 /+20$ & $\pm 35 / 0$ & $20 / 4$ & $0.40 / 0.29$ \\
\hline & 2 & $3 / 0.67$ & $0.5 / 0.33$ & & & & & & & \\
\hline \multirow[t]{2}{*}{2} & 3 & $3 / 0.07$ & $0.5 / 0.22$ & \pm 0.00004 & $\pm 0.02 \%$ & $5 / 3$ & $\pm 25 /+9$ & $\pm 35 /-13$ & $20 / 3$ & $0.40 / 0.25$ \\
\hline & 4 & $3 / 0.62$ & $0.5 / 0.16$ & & & & & & & \\
\hline \multirow[t]{2}{*}{3} & 6 & $3 / 1.95$ & $0.5 / 0.16$ & \pm 0.00004 & $\pm 0.02 \%$ & $10 / 5$ & $\pm 50 /-10$ & $\pm 35 /+18$ & $20 / 9$ & $0.40 / 0.32$ \\
\hline & 7 & $3 / 1.50$ & Asp & & & & & & & \\
\hline \multirow[t]{2}{*}{4} & 8 & $3 / 1.20$ & $0.5 / 0.43$ & \pm 0.00004 & $\pm 0.02 \%$ & $5 / 3$ & $\pm 25 /+23$ & $\pm 35 /-13$ & $20 / 2$ & $0.40 / 0.29$ \\
\hline & 9 & $3 / 1.70$ & $0.5 / 0.23$ & & & & & & & \\
\hline \multirow[t]{2}{*}{5} & 10 & $3 / 0.00$ & $0.5 / 0.14$ & \pm 0.00004 & $\pm 0.02 \%$ & $5 / 5$ & $\pm 25 /+18$ & $\pm 35 /+3$ & $20 / 3$ & $0.40 / 0.38$ \\
\hline & 11 & $3 / 2.00$ & $0.5 / 0.25$ & & & & & & & \\
\hline \multirow[t]{2}{*}{6} & 12 & $3 / 1.50$ & Asp & \pm 0.00004 & $\pm 0.02 \%$ & $10 / 5$ & $\pm 50 /-36$ & $\pm 35 /-23$ & $20 / 1$ & $0.40 / 0.05$ \\
\hline & 13 & $3 / 1.80$ & $0.5 / 0.20$ & & & & & & & \\
\hline \multirow[t]{2}{*}{7} & 14 & $3 / 1.50$ & $1 / 0.46$ & \pm 0.00004 & $\pm 0.02 \%$ & $5 / 0$ & $\pm 25 /+13$ & $\pm 35 / 0$ & $20 / 7$ & $0.40 / 0.15$ \\
\hline & 15 & $3 / 0.07$ & $1 / 0.07$ & & & & & & & \\
\hline
\end{tabular}

ETD - maximum edge thickness minus minimum edge thickness (TIR)

Fringes power and irregularity - difference from test plate @ 632 nm

$\mathrm{dN}$ - refractive index difference, $\mathrm{dV}$ - Abbe number change

Surface 7 Asphere Tolerance: less than 0.07 microns peak to valley over any $14 \mathrm{~mm}$ diameter subaperture (RRU met spec).

Surface 12 Asphere Tolerance: less than 0.05 microns peak to valley over any $17 \mathrm{~mm}$ diameter subaperture (RRU met spec). 


\section{Expected BPFF15 with tolerances}

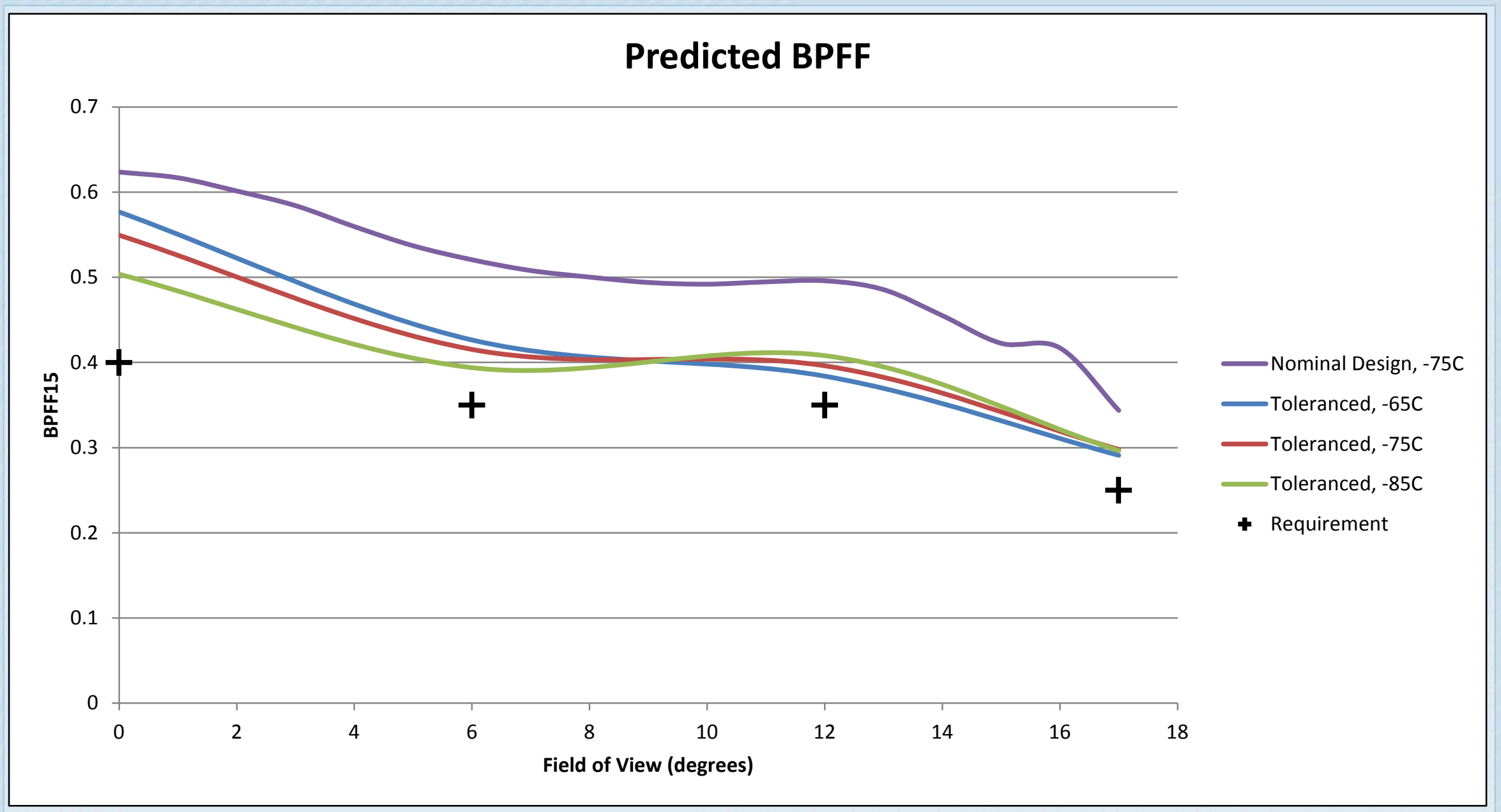

- Expected performance (84\% probability) 


\section{Fabrication and Alignment Procedure}

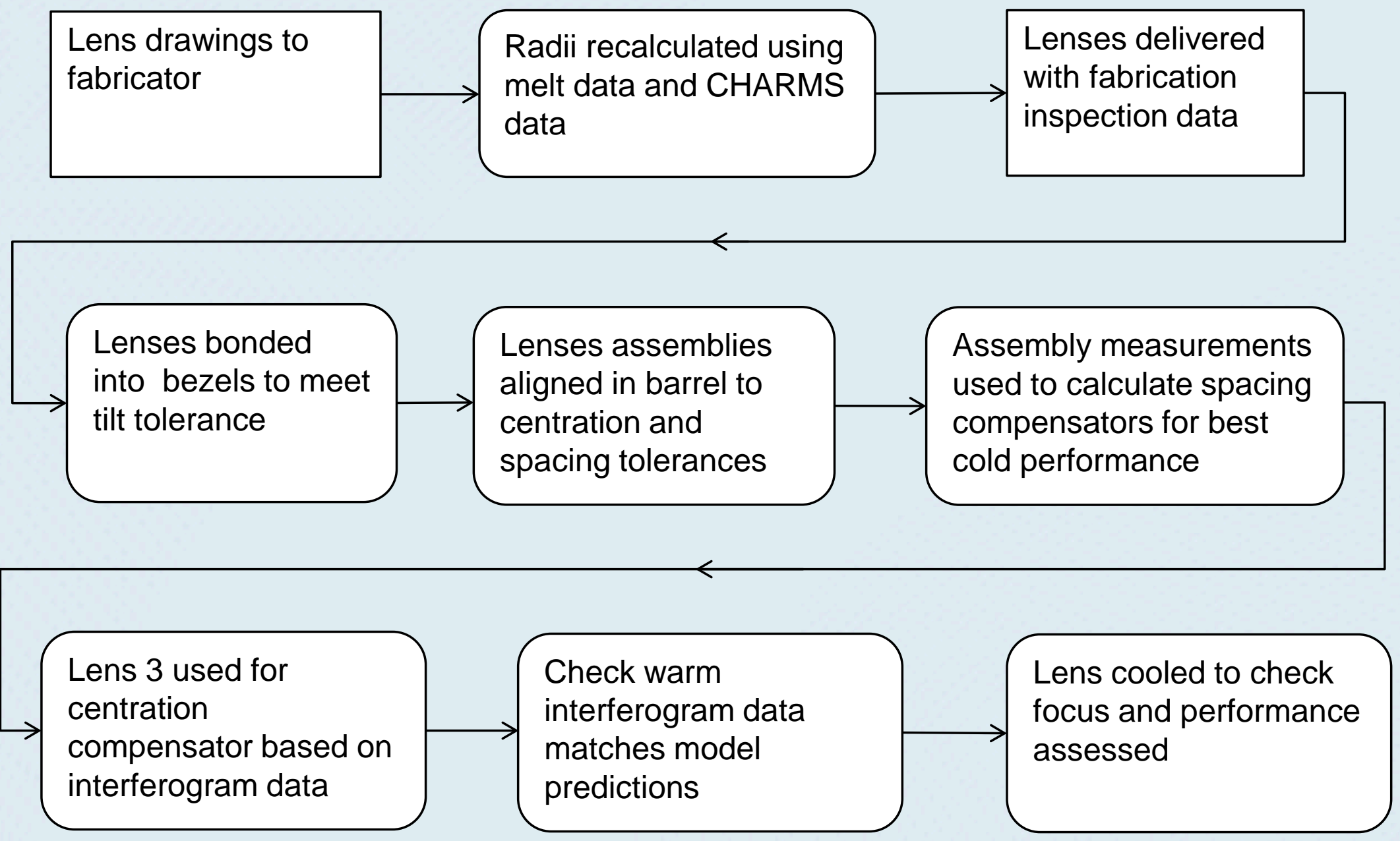


- Catalog index and dispersion data does not extend to $-75 \mathrm{C}$

- NASA Goddard Cryogenic, High Accuracy Refraction Measuring System (CHARMS) capable of measuring refractive index of materials down to $10 \mathrm{~K}$

Cryogenic Refractive Indices of S-LAH55, S-LAH55V, S-LAH59, SLAM3, S-NBM51, S-NPH2, S-PHM52, and S-TIH14 Glasses

Kevin H. Miller, ${ }^{1}$ Manuel A. Quijada, and Doug Leviton

Goddard Space Flight Center, 8800 Greenbelt Road Greenbelt, MD 20771;

\section{ABSTRACT}

The Transiting Exoplanet Survey Satellite (TESS) is an explorer-class planet finder, whose principal goal is to detect small planets with bright host starts in the solar neighborhood. The TESS payload consists of four identical cameras with seven optical elements each that include various types of Ohara glass substrates. The successful implementation both panchromatic and thermal lens assembly designs for these cameras requires a fairly accurate (up to 1E-6) knowledge of the temperature and wavelength dependence of the refractive index in the wavelength and temperature range of operation. Hence, this paper is devoted to report on measurements of the refractive index over the wavelength range of $0.42-1.15$ um and temperature range of $110-310 \mathrm{~K}$ for the following Ohara glasses: S-LAHS5, S-LAHDSV, SLAH59, S-LAM3, S-NBMS1, S-NPH2, S-PHM52, and S-TIH14. The measurements were performed utilizing the Cryogenic High Accuracy Refraction Measunng System (CHARMS) faclity at NASA's Goddard Space Flight Center A dense coverage of the absolute refractive index for the title substrates in the aforementioned wavelength and temperature ranges was used to determine the thermo-optic coefficient $(\mathrm{dn} / \mathrm{dT})$ and dispersion relation $(\mathrm{dn} / \mathrm{d} \lambda)$ as a function of wavelength and temperature. A comparison of the measured indices with literature values, specifically the temperature-dependent refractive indices of S-PHMS2 and S-TIH14, will be presented.

Keywords: S-LAH55, S-LAH55V, S-LAH59, S-LAMB, S-NBM51, S-NPH2, S-PHM52, S-TIH14, CHARMS, cryogenic refractive index
Initial lens design, Ohara glass catalog at 20C

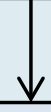

Insert a private glass catalog with CHARMS index data at $-75 \mathrm{C}$

Add zoom position for $-75 \mathrm{C}$ coupled using CTE and CHARMS data

Optimize radii and aspheric coefficients for cold performance. Maintain frozen center thicknesses and other design constraints.

Final design optimized for $-75 \mathrm{C}$, with zoom coupled to room-temperature radii for fabrication drawings.

Evaluate performance at $-65 \mathrm{C}$ to $-85 \mathrm{C}$ 


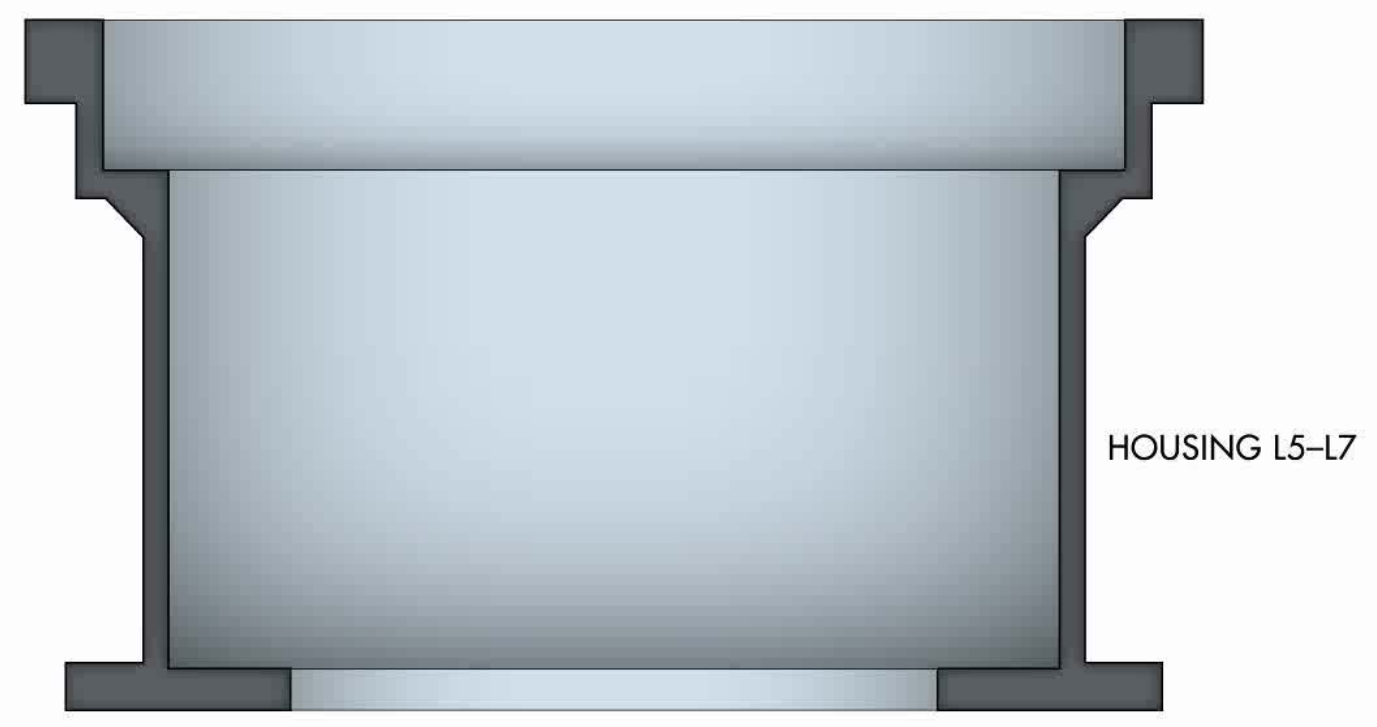


- RRU Lens Assembly Build - Pathfinder for Flight Build

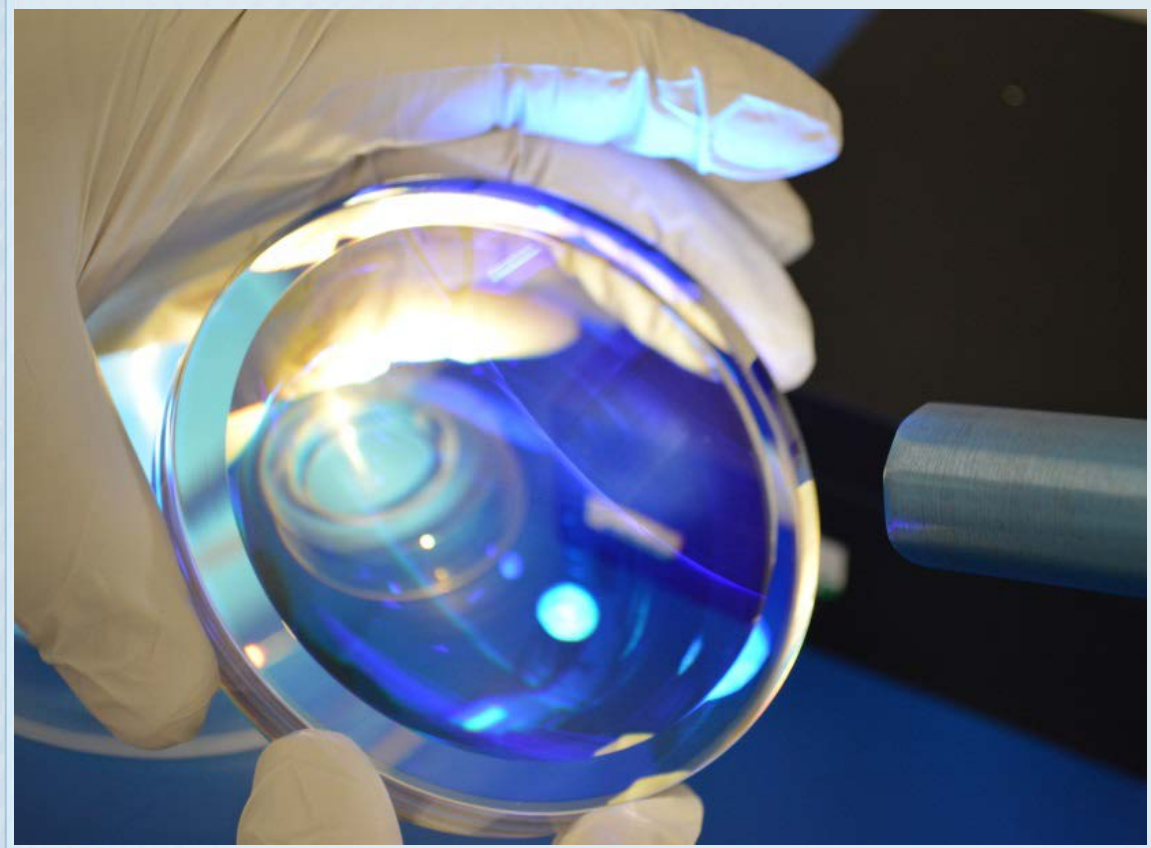

Lens Inspection

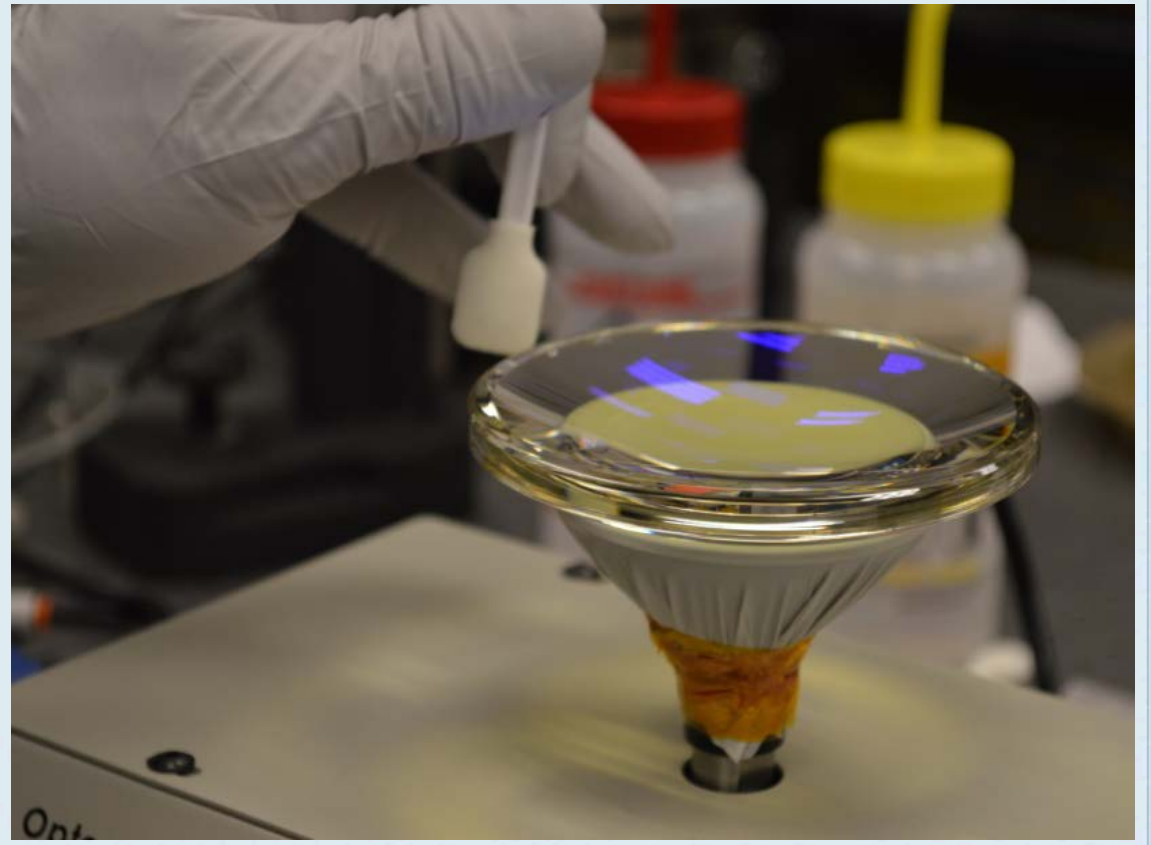

Lens Edge Preparation 


\section{Cess}

\section{Lens Build: RTV Pad Fabrication}

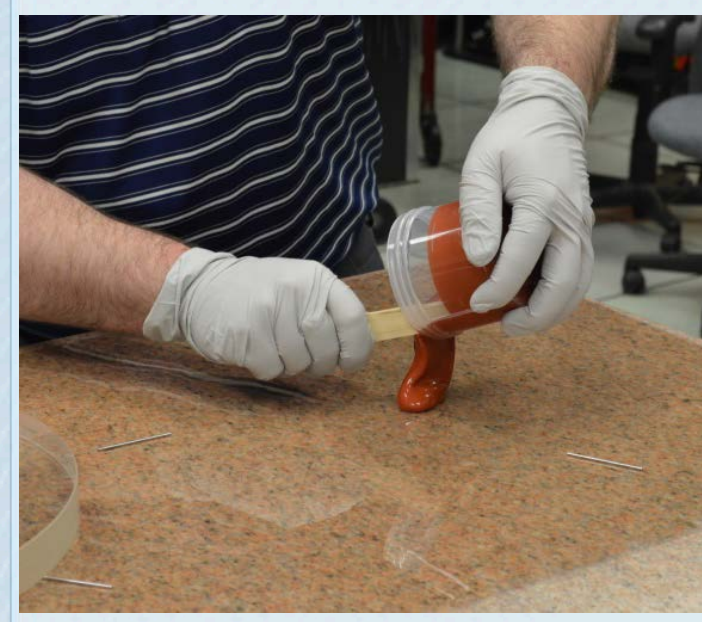

Mix

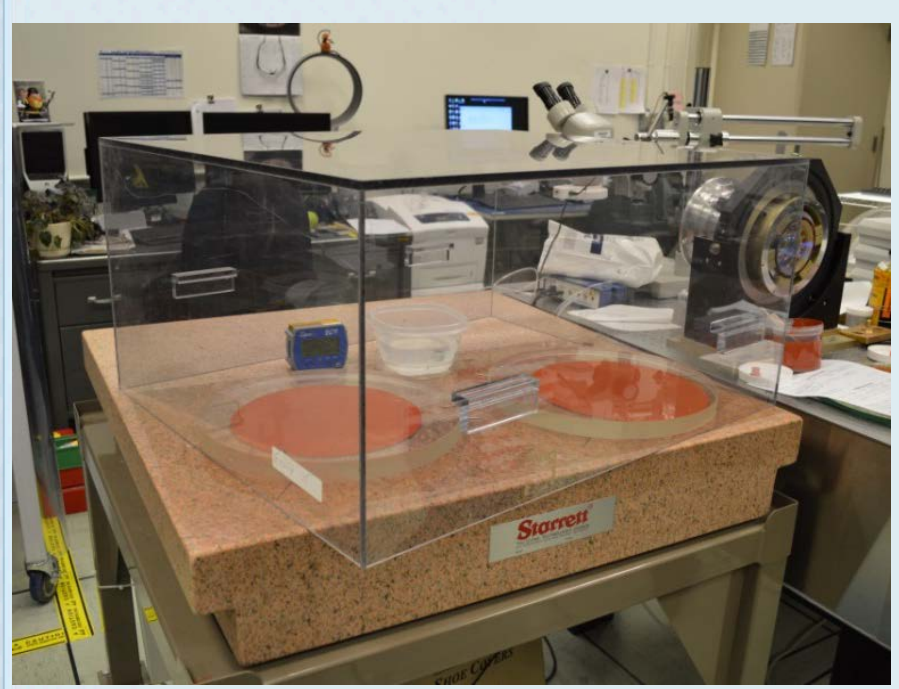

Cure

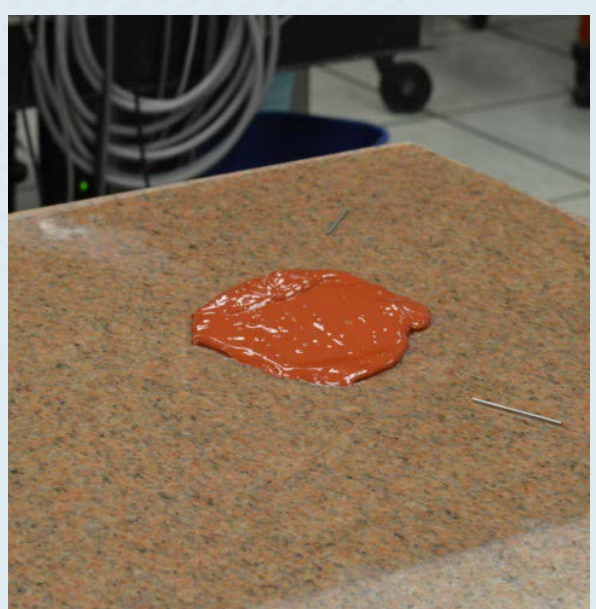

Pour

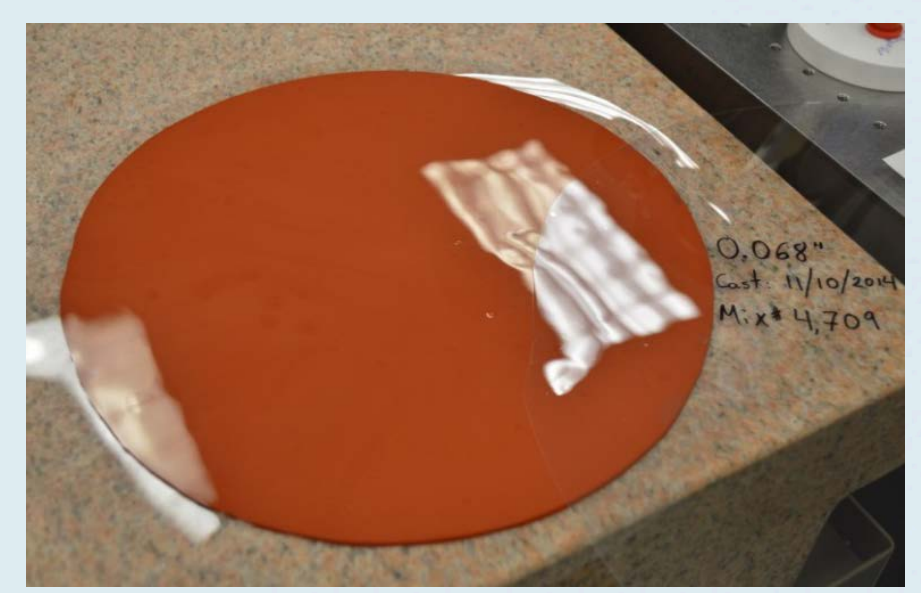

Cut

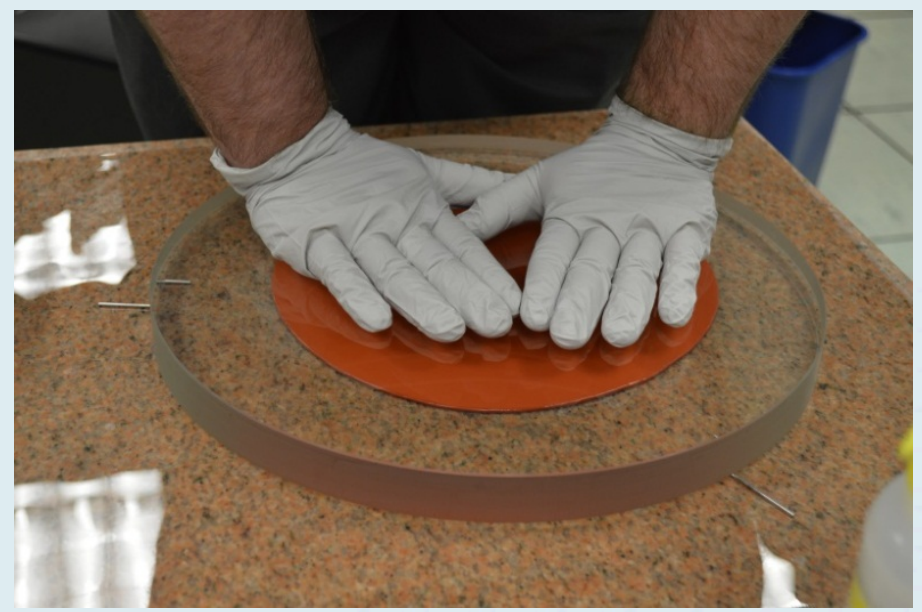

\section{Press}
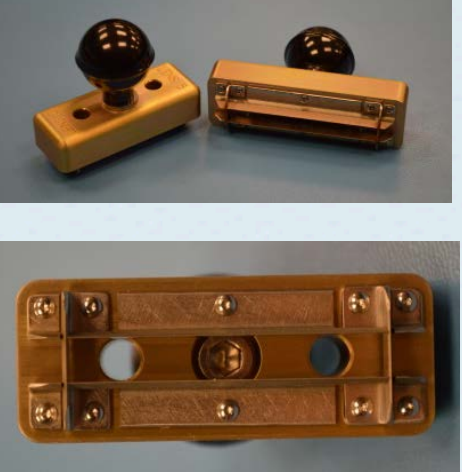


\section{Dess}

\section{Lens Build: Lenses With RTV Pads}

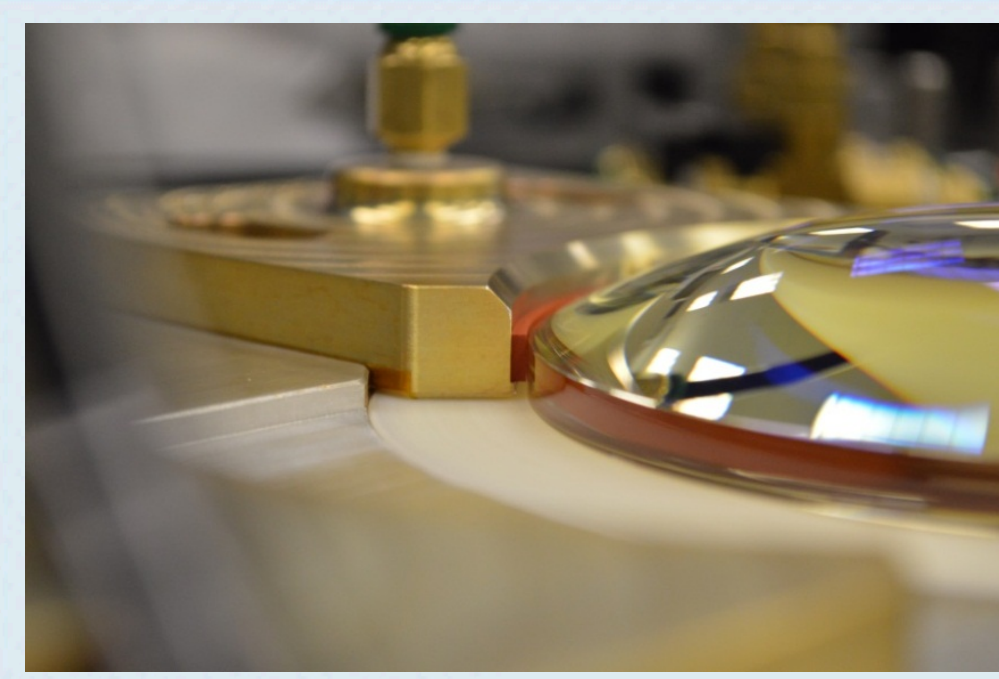

Fit Check



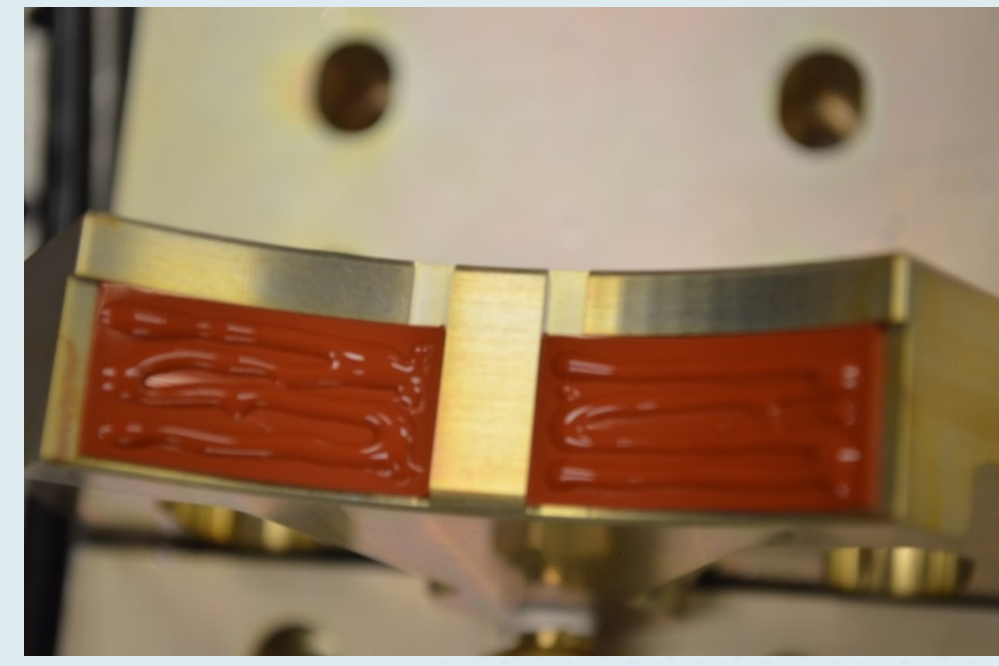

Pad Prep

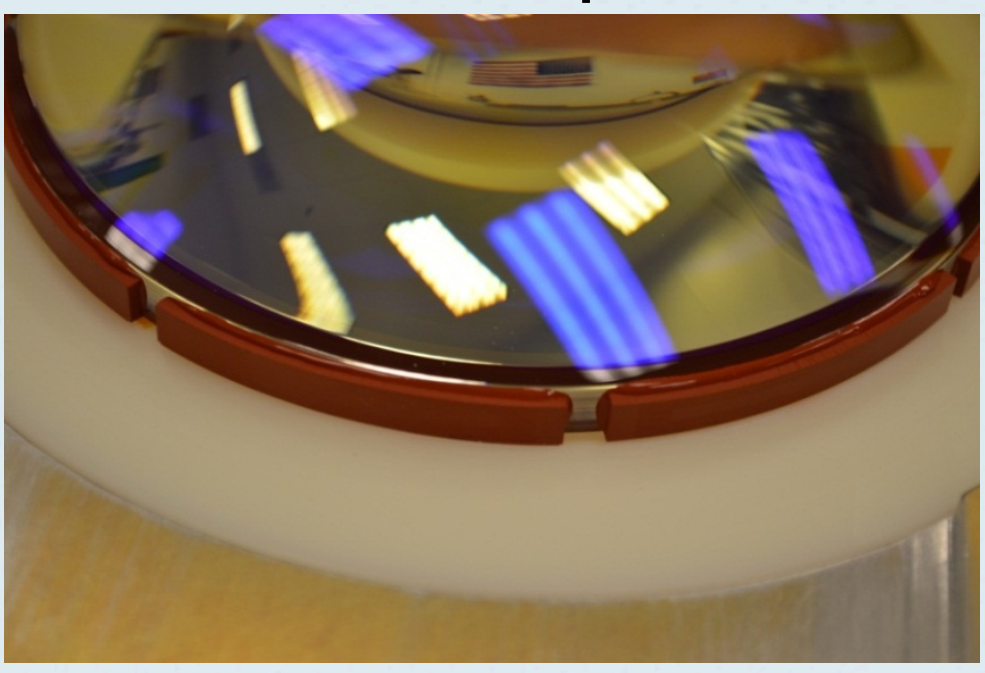

Pads on Lens 


\section{Dess}

\section{Lens Build: Lenses in Bezels}

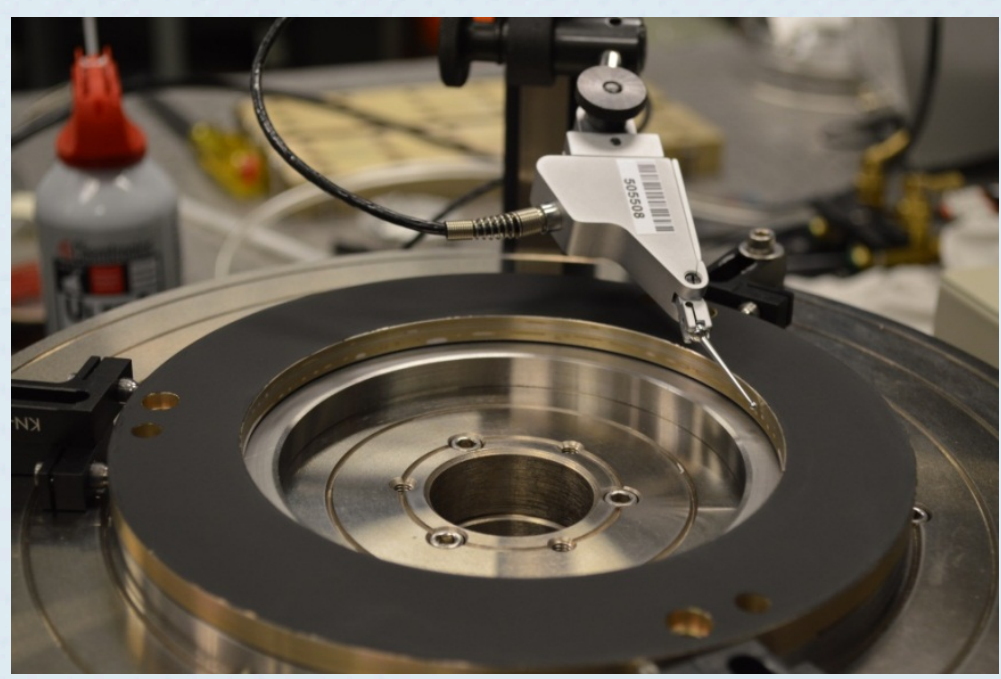

Bezel Check

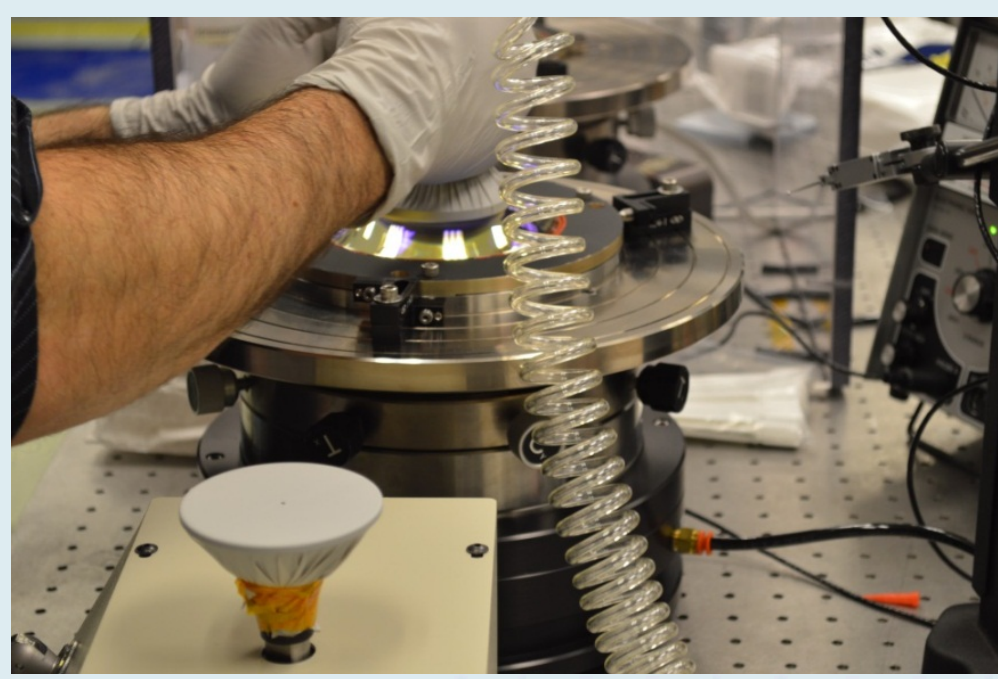

Lens Placement

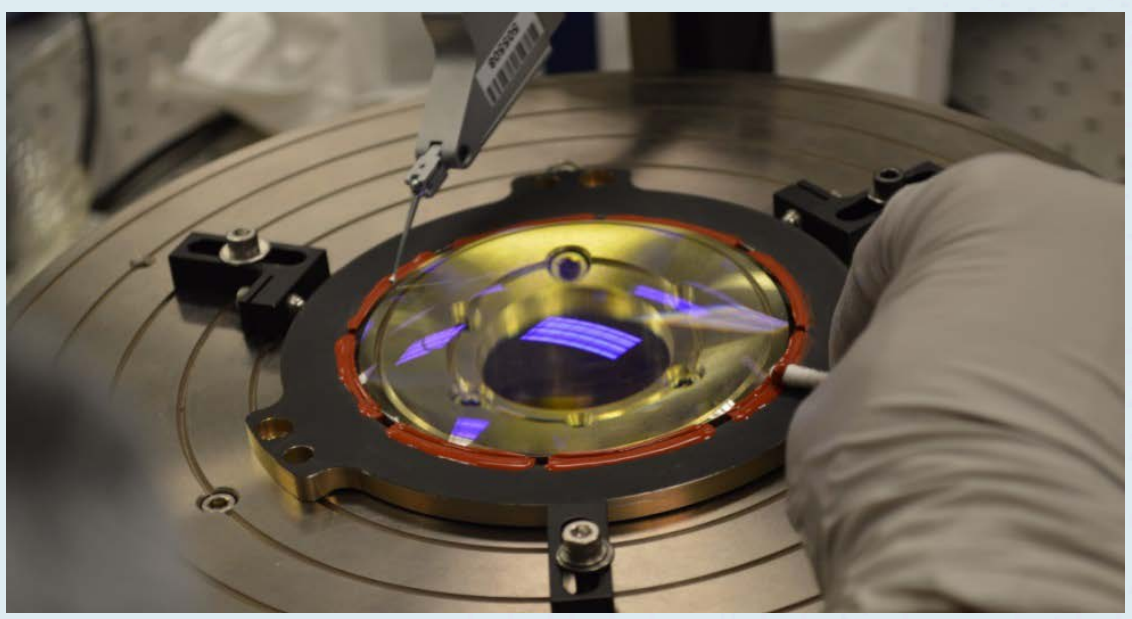

Lens in Bezel Runout 


\section{Eess}

\section{Lens Build: Lens Thermal Cycling}

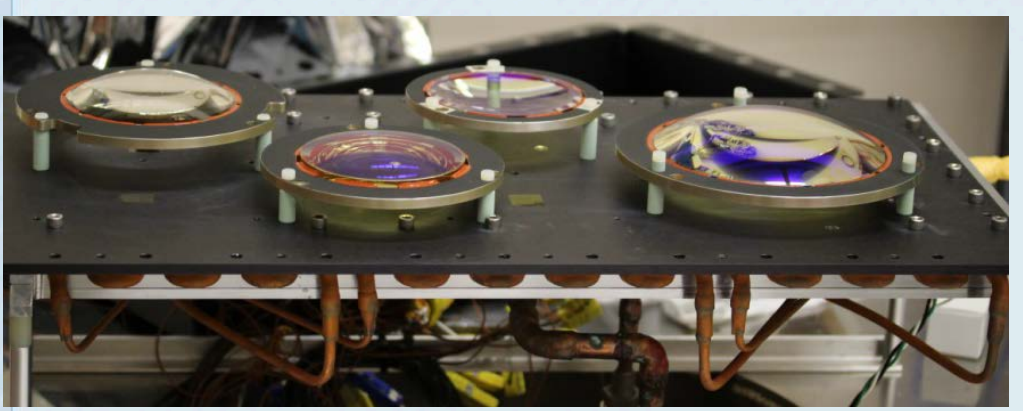

L1, L2, L3, L4 Mounted for Test

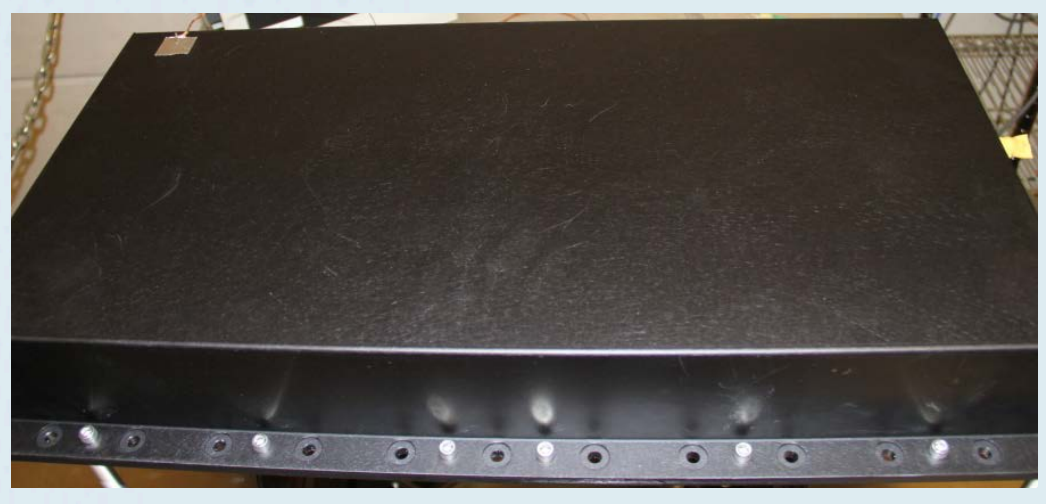

Cover (Dog-House) Installed

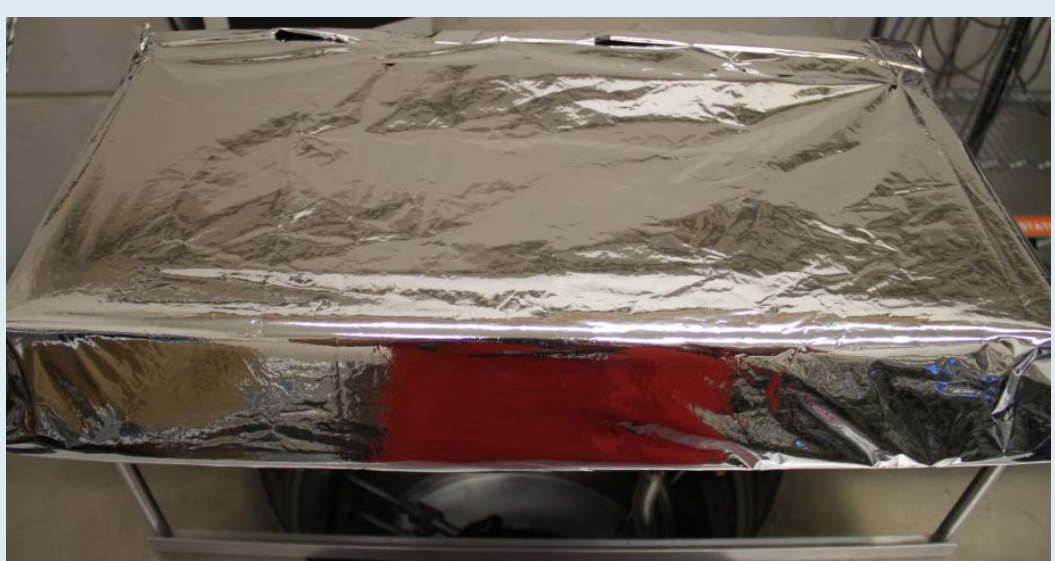

Thermal Blankets

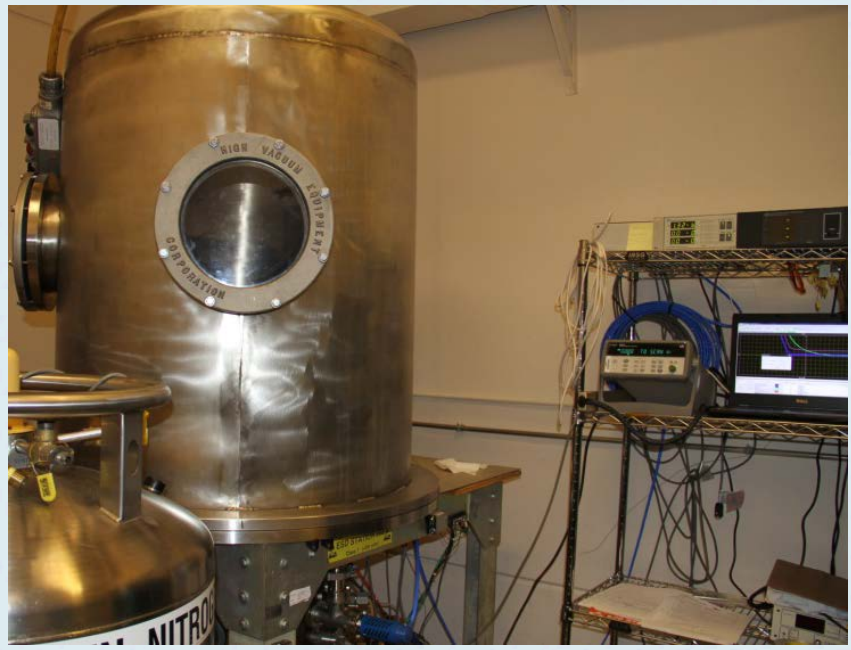

Thermal Chamber 
- Trioptics Lens Assembly Station

- Non-contact measurement of lens alignments

- Procedure details developed during RRU build

- Uncertainty in lens alignment: 2 microns in decenter and 1 micron axial displacement
Install shims and lens-bezel assy.
Center lens using pushers and optical feedback

Torque fasteners while monitoring centration
Measure lens axial position, decenter, and tilt

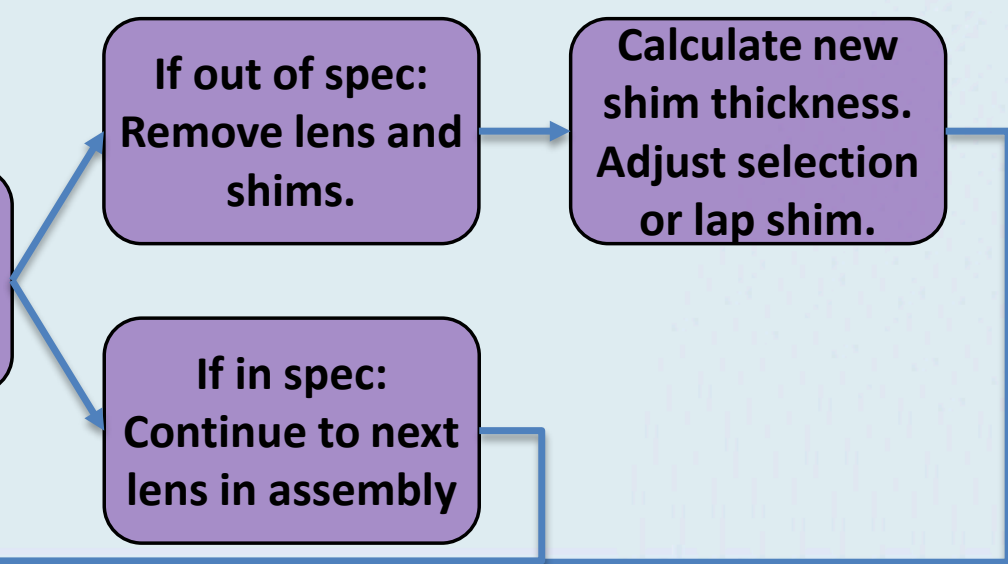

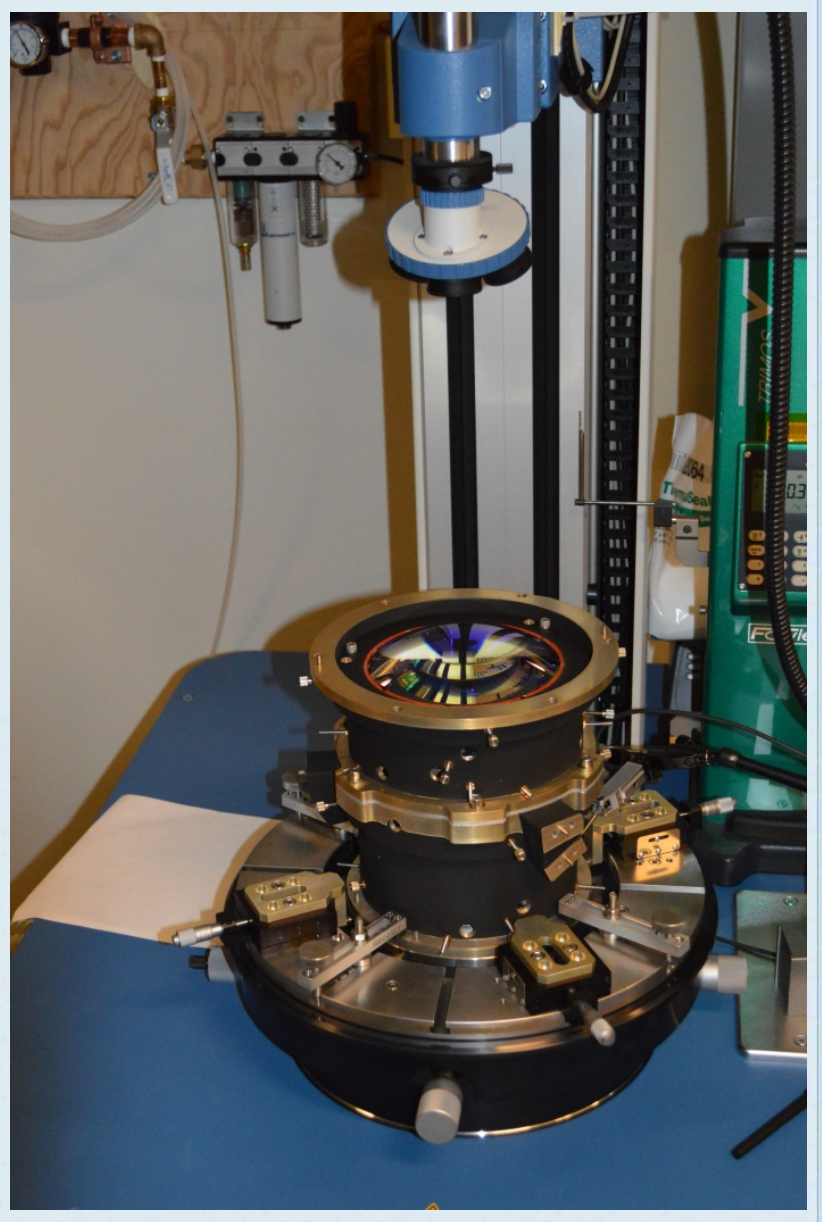




\section{Coss Lens Build: Barrel Assembly and Alignment}

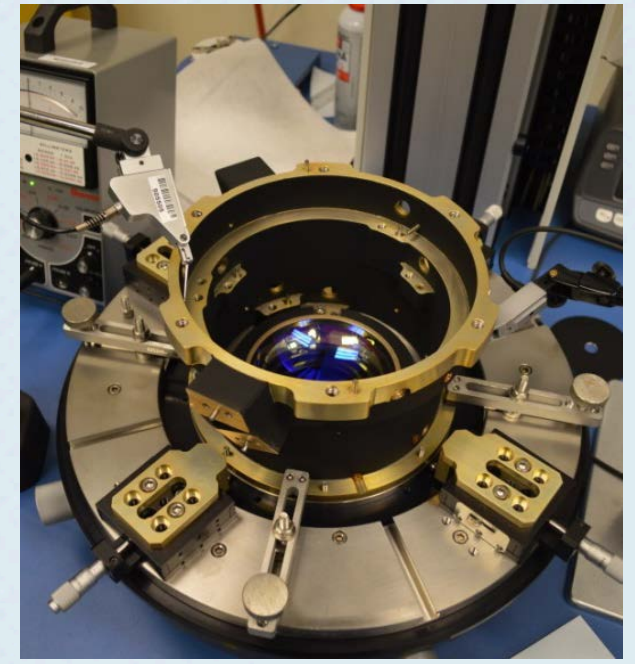

Lens install

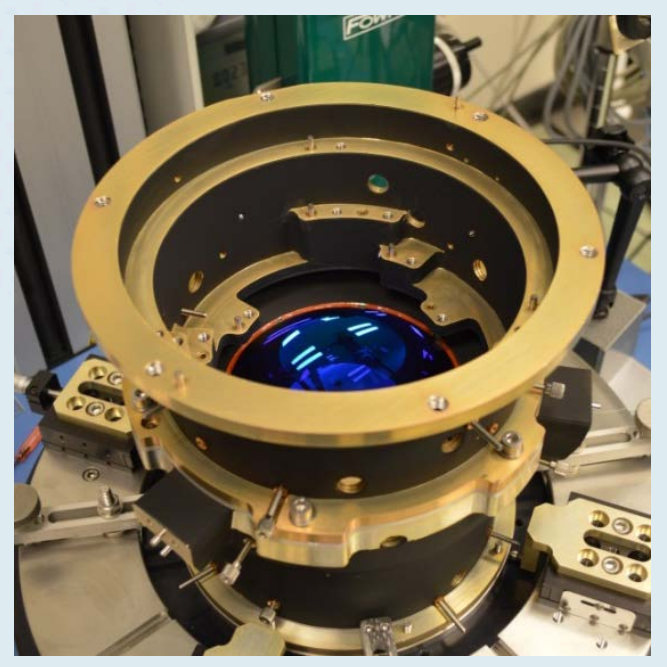

Upper Barrel

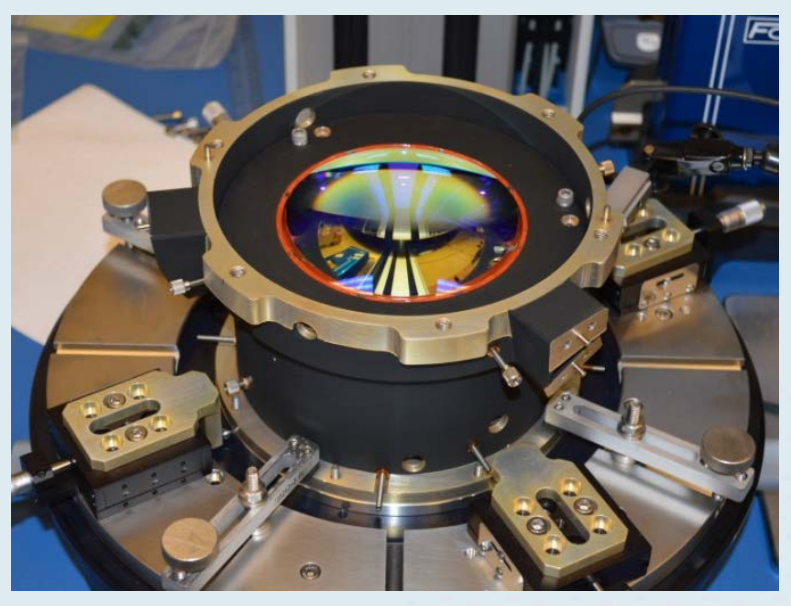

Lower Barrel Complete

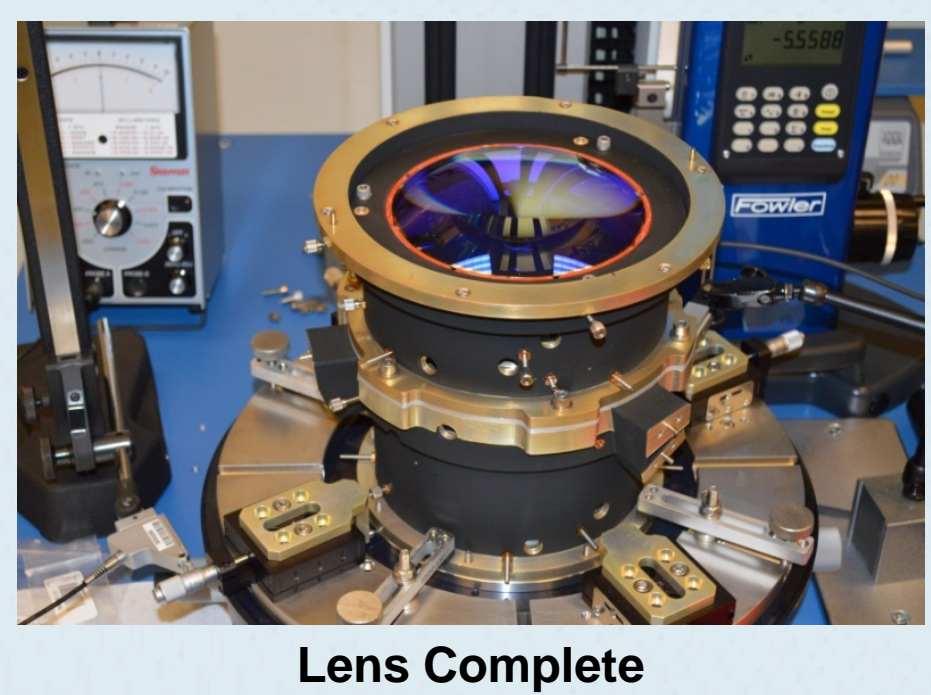




\section{TESS Optical Test}

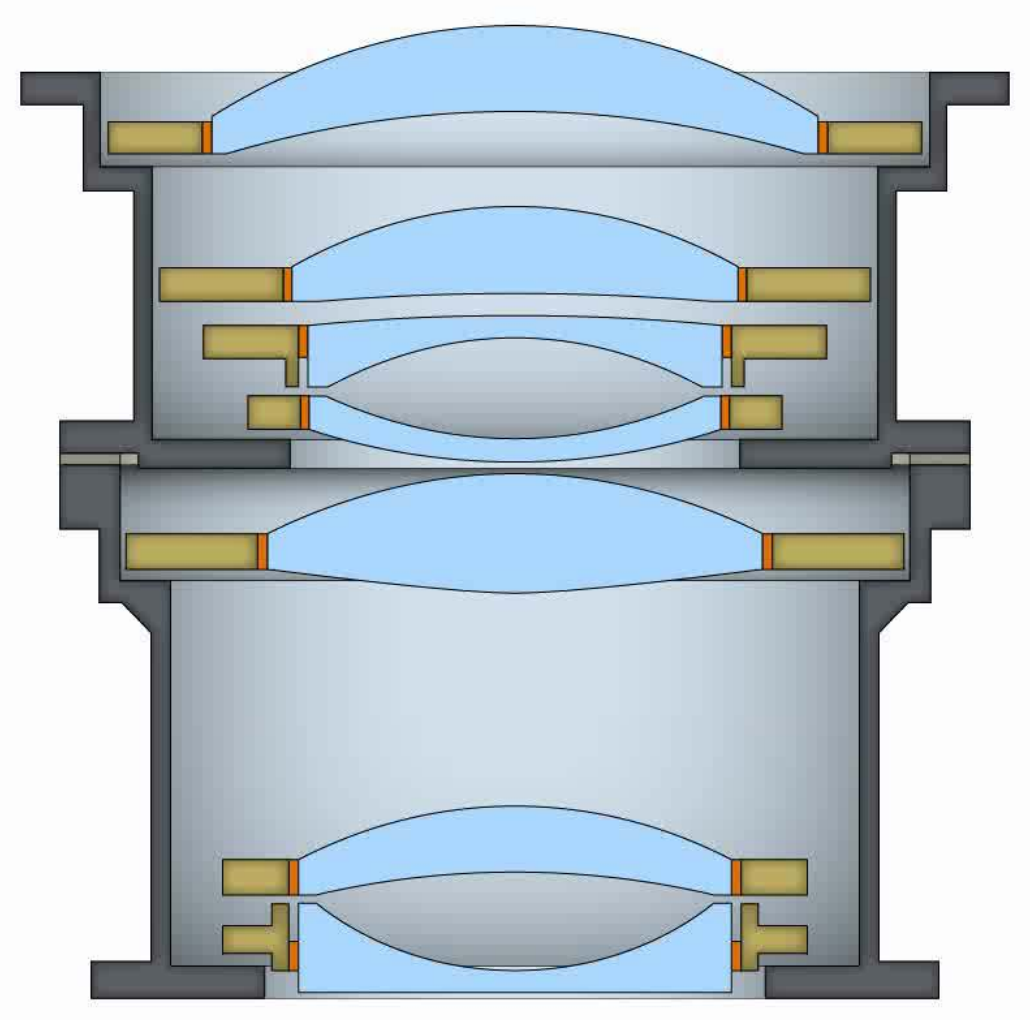


- Focus interferometer at focus of TESS, and return using large flat in object space

- Translate interferometer along focal plane, and follow return with large flat tilt

- Track interferometer location with respect to TESS lens, and track return flat angle with theodolite

- Take interferometric data across FOV and through focus

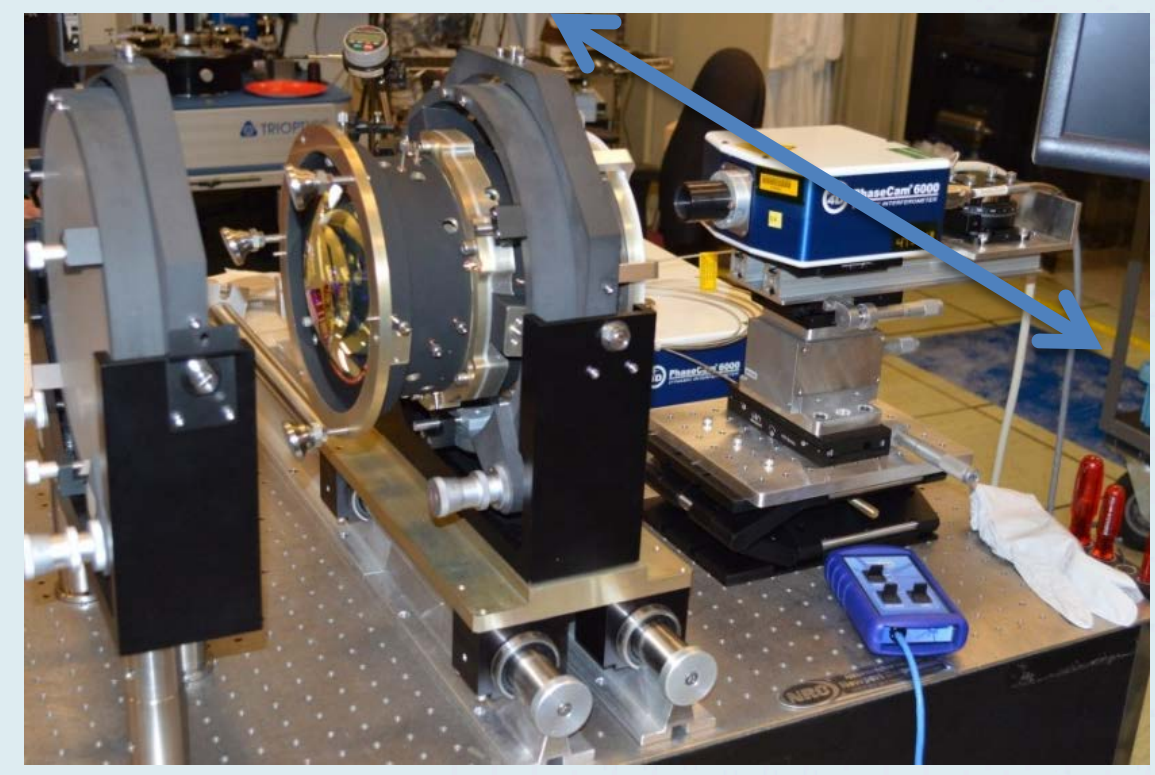


On axis, $\pm 10 \mathrm{~mm}\left(3.9^{\circ}\right)$ Wavefront Maps

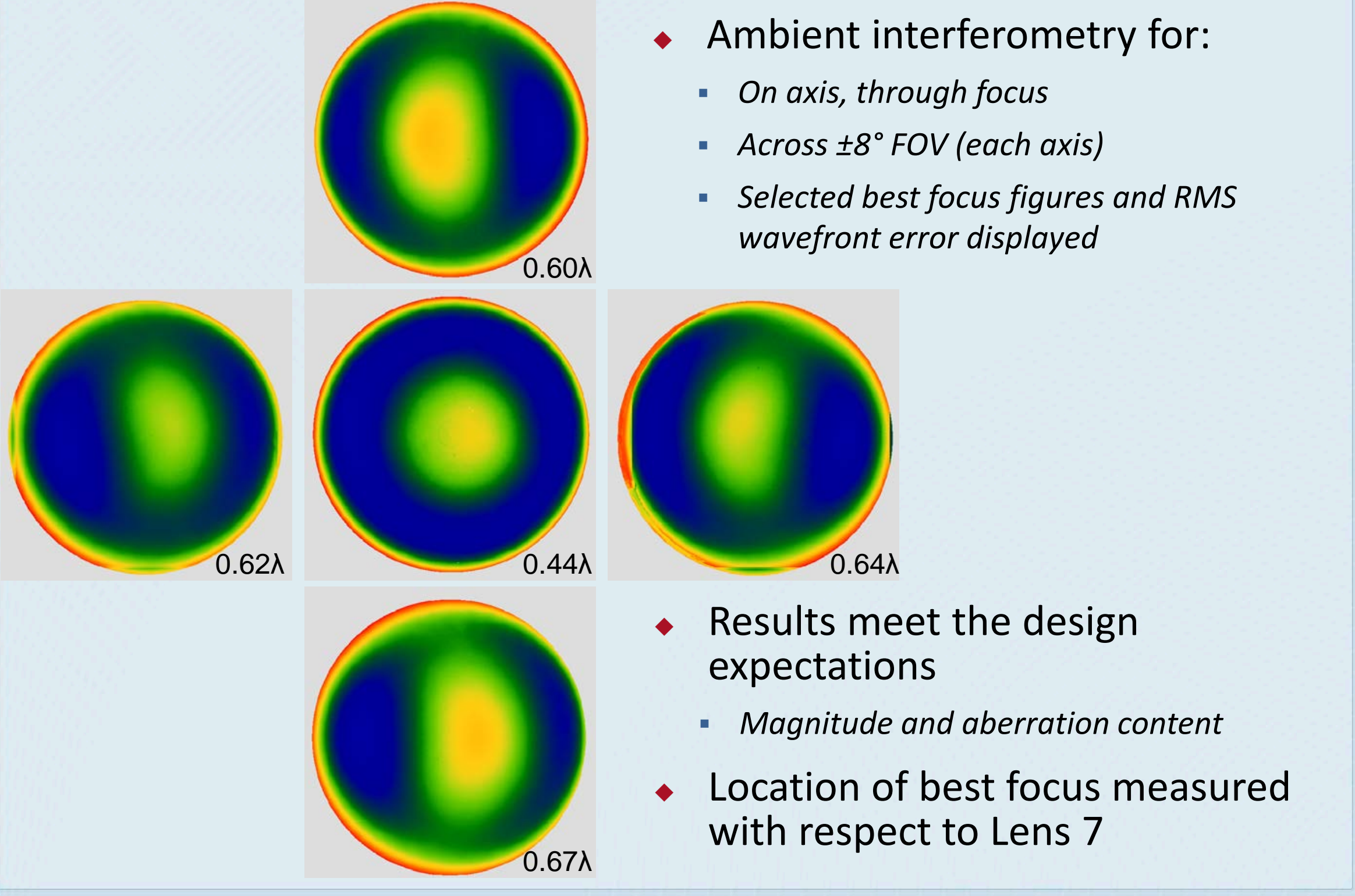




\section{- CODE V 'ALI' used to calculate compensator adjustments}

- Lens 1 and Focal Plane positions

Focal Plane Only

\begin{tabular}{|c|c|c|c|c|c|c|c|}
\hline \multicolumn{7}{|c|}{ Wavelength $=632.8 \mathrm{~nm}$. } & \\
\hline Zoom & Field & weight & of rays & pre-align po & post-align & & \\
\hline 1 & 1 & 1.000 & 7796 & 1.7191 & 0.3162 & & \\
\hline 1 & 2 & $1.00 \odot$ & 7501 & 1.2751 & 0.3609 & & \\
\hline 1 & 3 & 1.000 & 7397 & 1.2765 & 0.3555 & & \\
\hline 1 & 4 & 1.000 & 7733 & 1.5489 & 0.2716 & & \\
\hline 1 & 5 & 1.000 & 7665 & 1.5772 & 0.2664 & & \\
\hline 1 & 6 & 1.000 & 7175 & 1.4138 & 0.3829 & & \\
\hline 1 & 7 & 1.000 & 7421 & 1.4722 & 0.3229 & & \\
\hline 1 & 8 & 1.000 & 7580 & 1.5710 & 0.1865 & & \\
\hline 1 & 9 & 1.000 & 7520 & 1.4906 & 0.3257 & & \\
\hline 1 & 10 & 1.000 & 7766 & 1.7190 & 0.2714 & & \\
\hline 1 & 11 & 1.000 & 7765 & 1.7415 & 0.2596 & & \\
\hline 1 & 12 & 1.000 & 7414 & 1.6074 & 0.3016 & & \\
\hline 1 & 13 & 1.000 & 7505 & 1.5784 & 0.2160 & & \\
\hline \multicolumn{2}{|c|}{ TOTAL } & & & 1.5470 & 0.2995 & & \\
\hline \multirow{2}{*}{\multicolumn{4}{|c|}{$\begin{array}{l}\text { Compensator } \\
\text { type }\end{array}$}} & Compensator & or $\quad$ RMS (a) & RMS (b) & \\
\hline \multirow{2}{*}{\multicolumn{4}{|c|}{ * DLZ S16 }} & value & difference & contribution & \\
\hline & & & & $\begin{array}{l}0.45941 E-01 \\
-.68563 E-04\end{array}$ & 0.3055 & $\begin{array}{l}1.5177 \\
0.9220\end{array}$ & $\begin{array}{l}\text { (focus) } \\
\text { (image }\end{array}$ \\
\hline \multicolumn{2}{|l|}{ tilt) } & & & $-.68563 E-04$ & & & \\
\hline & $\star \star$ DLB & & $0.17755 E-03$ & 1.5470 & 0.0562 & (image \\
\hline
\end{tabular}

tilt) (a) TOTAL RMS wavefront difference assuming only one compensator is active

(b) TOTAL RMS wavefront error introduced by the one compensator
Focal Plane and Lens 1

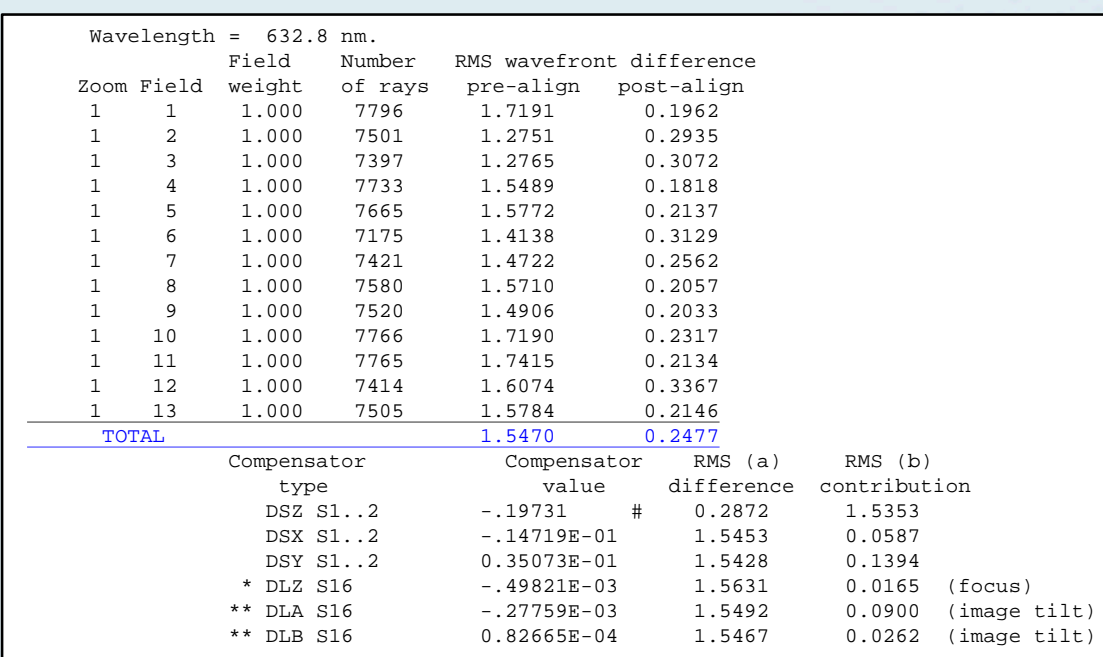

(a) TOTAL RMS wavefront difference assuming only one compensator is active

(b) TOTAL RMS wavefront error introduced by the one compensator

\# Compensator has been damped to avoid exceeding maximum value
Did not adjust Lens 1 since performance predictions met

BPFF specification with only focus adjustment. 


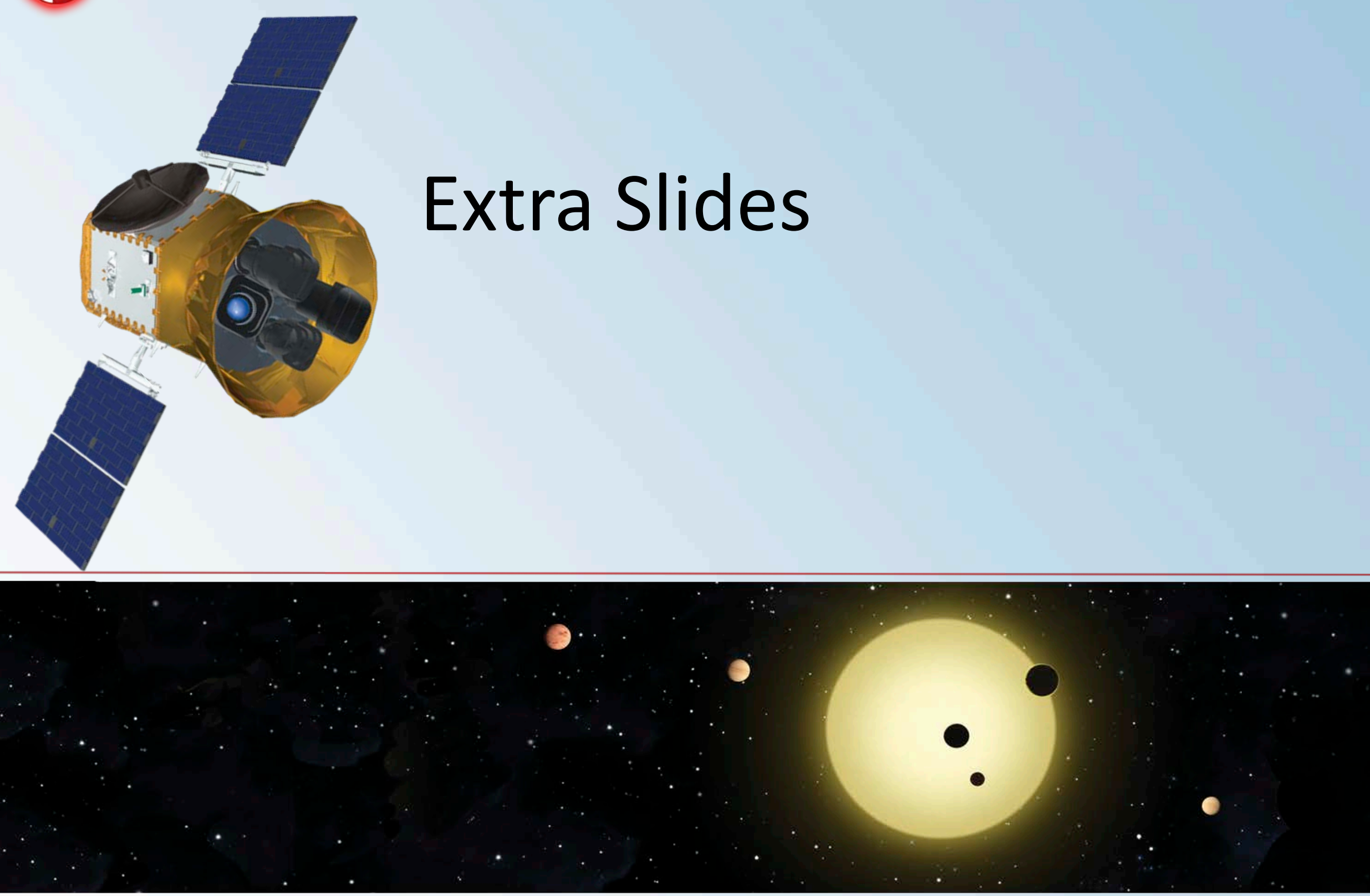

This work is sponsored by the National Aeronautics and Space Administration under Air Force Contract \#FA8721-05-C-0002. Opinions, interpretations, conclusions and recommendations are those of the author and are not necessarily endorsed by the United States Government. 


\section{TESS Detection Goals and Target Stars}

- Discover transiting earths and super earths

- Orbiting bright, nearby stars

- Rocky planets and water worlds

- Habitable planets

Habitable Zone

Transits/year

Hotter Stars

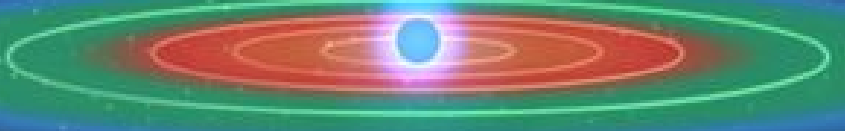

$<<1$

Sun-like Stars

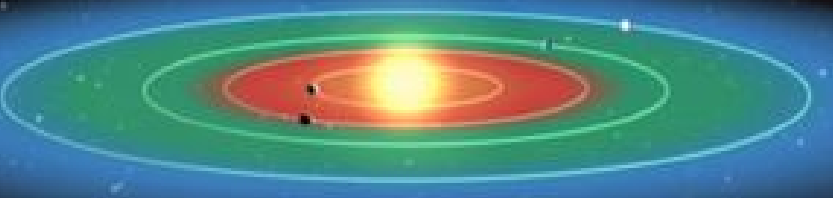

Cooler Stars

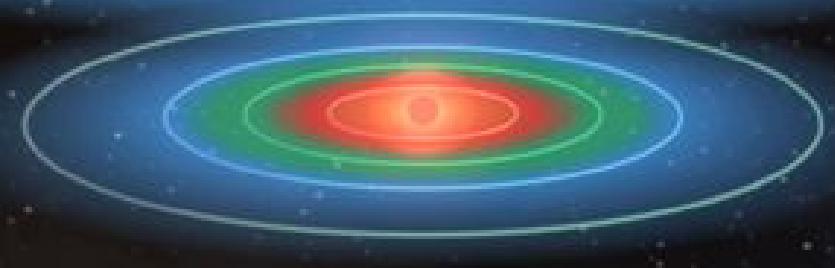

$\gg 1$ 
- With a 2 year mission duration, TESS is expected to discover:

- 30 Earth-sized planets

- $10-20 \%$ inside the habitable zone

- 20-30\% inside JWST's Continuous Viewing Zone

- 300 Super-Earth $\left(2 \cdot R_{E}\right)$ planets

- Tens of thousands of larger planets
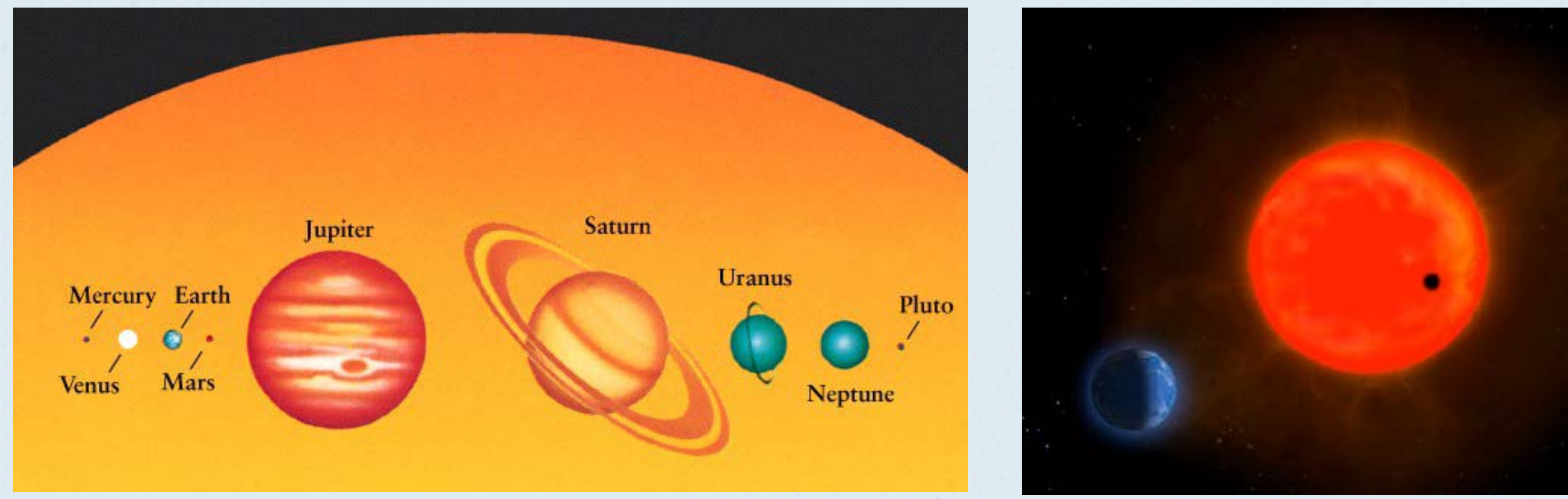


\section{Instrument Level Overview}

- TESS Instrument consists of 5 primary mechanical subsystems:
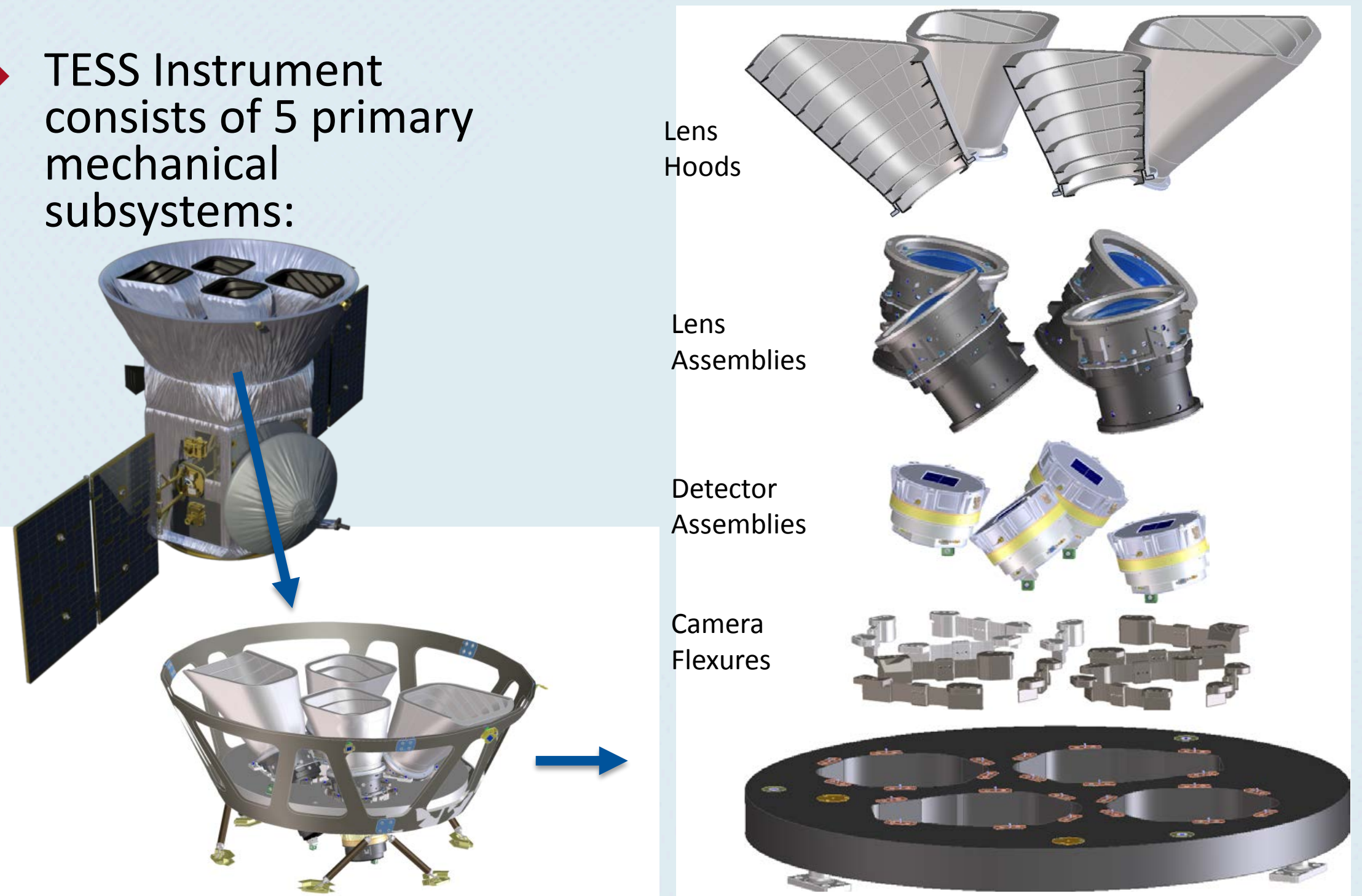
Thermal to Structural Temperature Mapping



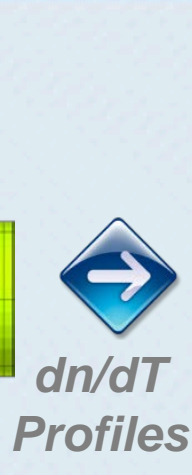

Surface Distortion Rigid Body Displacements

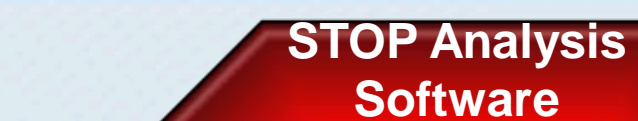

Software

Environment

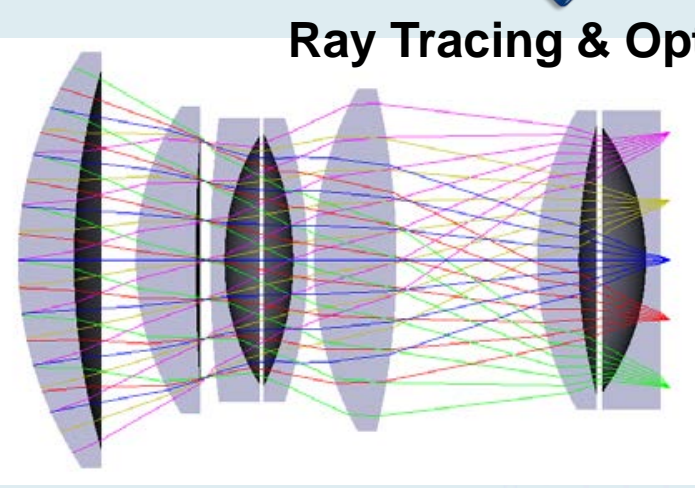

Structural Loading

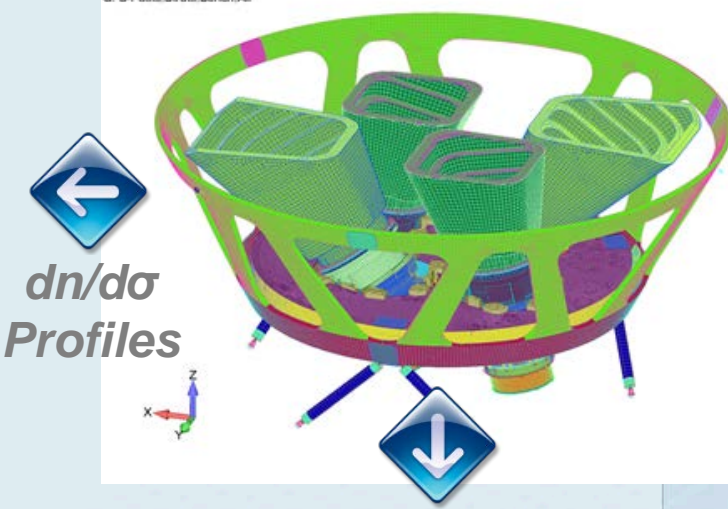

Structural Analysis
Surface Distortion

Rigid Body

Displacements

Ray Tracing \& Optical Analysis

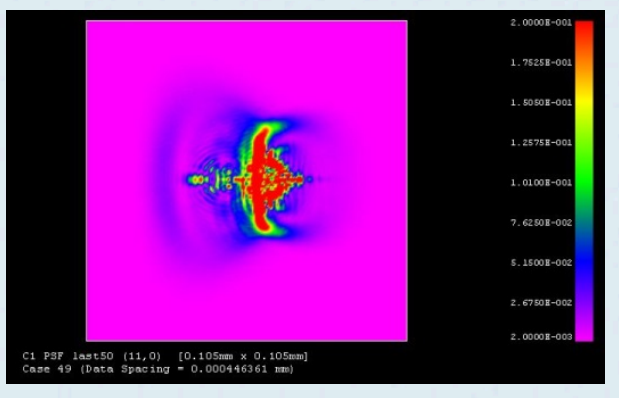




\section{Imaging Performance on Orbit}

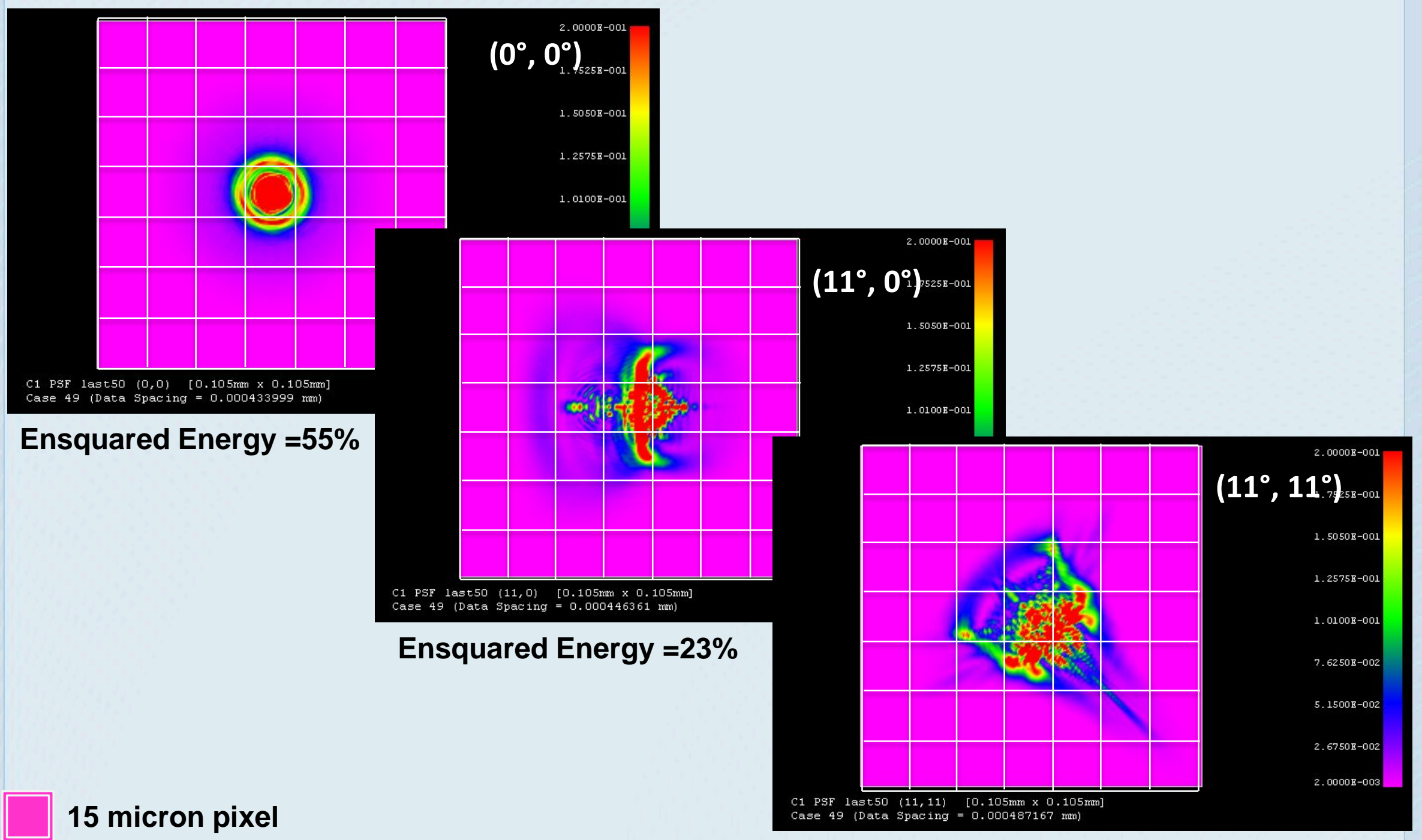

Ensquared Energy $=18 \%$ 


\begin{tabular}{|ll|}
\hline Parameter & Requirement \\
\hline CCD & $2 \times 2$ detector arrays \\
\hline Detector Array & $2048 \times 2048$ \\
\hline Pixel Size & 15 microns \\
\hline CCD Active Area Size & $63.482 \mathrm{~mm} \times 63.482 \mathrm{~mm}$ \\
\hline $\begin{array}{l}\text { CCD active area diagonal } \\
\text { semi-height }\end{array}$ & $44.88 \mathrm{~mm}$ \\
\hline
\end{tabular}




\section{TESS Imager Summary}

- $2048 \times 2048$ frame-transfer format, (2k x 4k CCD)

- $15-\mu \mathrm{m}$ pixels

\begin{tabular}{|c|c|c|}
\hline Performance & Value & Achieved \\
\hline Well Capacity & $>150,000$ e- (goal) & $>190,000 \mathrm{e}-$ \\
\hline Conversion Gain & $<10 \mu \mathrm{V} / \mathrm{e}-$ & $7 \mu \mathrm{V} / \mathrm{e}-$ \\
\hline $\begin{array}{l}\text { Read Noise } \\
@ 625 \text { kHz }\end{array}$ & $<20$ e- & $<14$ e- w/FPE \\
\hline $\begin{array}{r}\text { Dark Current } \\
@-30^{\circ} \mathrm{C}\end{array}$ & $<8 \mathrm{e}-/ \mathrm{pix} / \mathrm{sec}$ & $<2.5 \mathrm{e}-/ \mathrm{p} / \mathrm{s}$ \\
\hline Device Thickness & $\begin{array}{c}100 \mu m \\
(-10 /+15 \mu m)\end{array}$ & $95-115 \mu \mathrm{m}$ \\
\hline $\begin{array}{r}\text { Depletion-depth } \\
\text { control }\end{array}$ & Substrate bias & Functional \\
\hline $\begin{array}{r}\text { Targeted Spectral } \\
\text { Range }\end{array}$ & $600-1000 \mathrm{~nm}$ & 70\%@ @ 950 nm \\
\hline
\end{tabular}

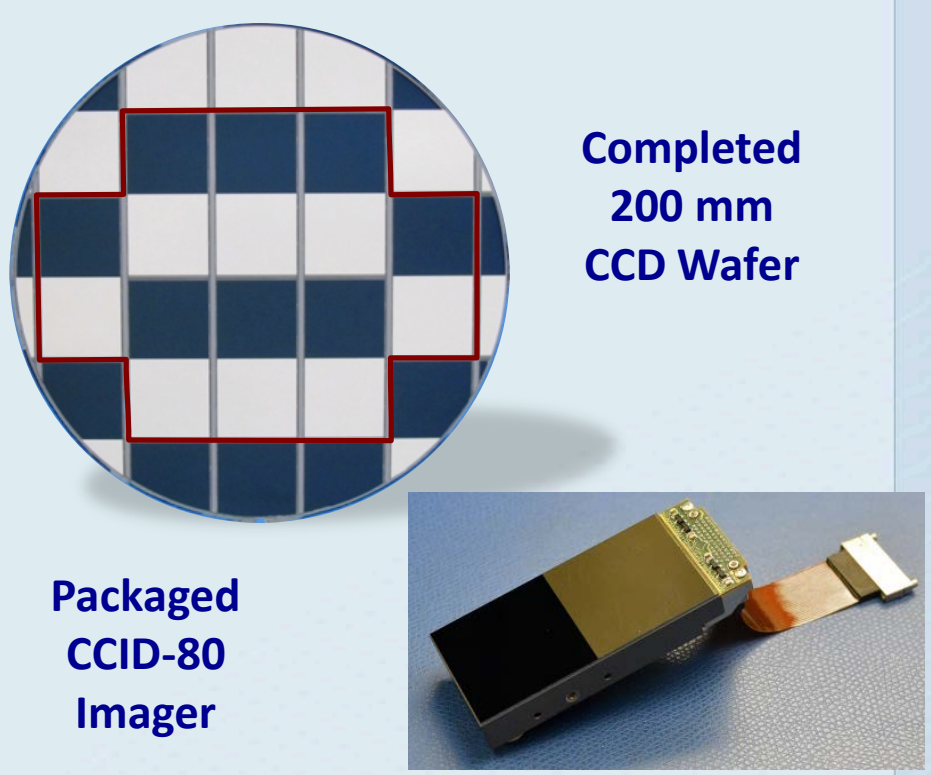

Prototype Detector Assembly

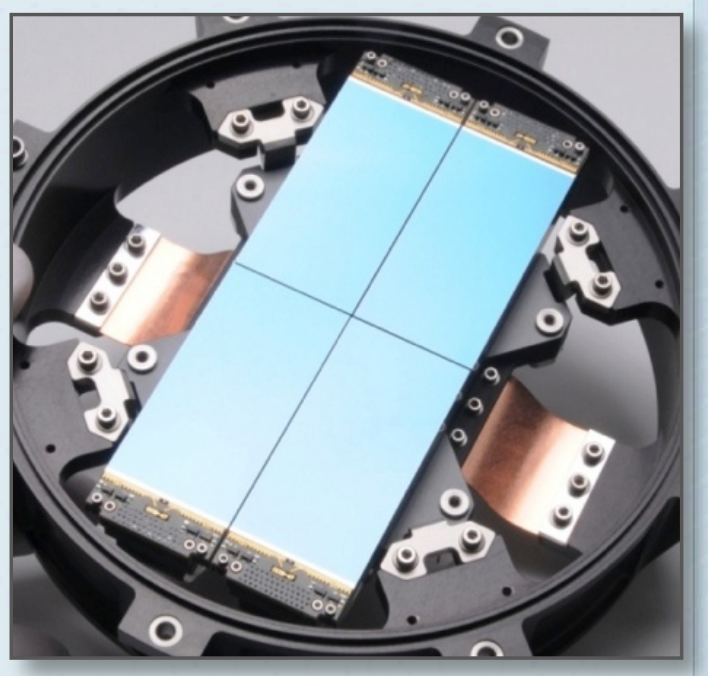




\section{Lens Assembly Design Tolerances}

\begin{tabular}{|c|c|c|c|c|c|c|c|c|c|c|}
\hline $\begin{array}{l}\mathrm{L} \\
\mathrm{e} \\
\mathrm{n} \\
\mathrm{s}\end{array}$ & Sur. & $\begin{array}{l}\text { Fringes } \\
\text { (power) }\end{array}$ & $\begin{array}{c}\text { Fringes } \\
\text { (irregularity) }\end{array}$ & $\mathbf{d N}$ & $d V$ & $\begin{array}{c}\text { Lens } \\
\text { wedge } \\
\text { (ETD mm) }\end{array}$ & $\begin{array}{l}\text { Lens } \\
\text { thickness } \\
\text { (mm) }\end{array}$ & $\begin{array}{l}\text { Axial } \\
\text { position } \\
\text { (mm) }\end{array}$ & $\begin{array}{c}\text { Radial } \\
\text { decenter } \\
\text { (mm) }\end{array}$ & $\begin{array}{l}\text { Lens tilt } \\
\text { (arc min) }\end{array}$ \\
\hline \multirow[t]{2}{*}{1} & 1 & 3 & 0.5 & \pm 0.00004 & $\pm 0.02 \%$ & \pm 0.005 & \pm 0.025 & \pm 0.035 & \pm 0.020 & \pm 0.4 \\
\hline & 2 & 3 & 0.5 & & & & & comp. & comp. & \\
\hline \multirow[t]{2}{*}{2} & 3 & 3 & 0.5 & \pm 0.00004 & $\pm 0.02 \%$ & \pm 0.005 & \pm 0.025 & \pm 0.035 & \pm 0.020 & \pm 0.4 \\
\hline & 4 & 3 & 0.5 & & & & & & & \\
\hline \multirow[t]{2}{*}{3} & 6 & 3 & 0.5 & \pm 0.00004 & $\pm 0.02 \%$ & \pm 0.010 & \pm 0.050 & \pm 0.035 & \pm 0.020 & \pm 0.4 \\
\hline & 7 & 3 & Asp & & & & & & & \\
\hline \multirow[t]{2}{*}{4} & 8 & 3 & 0.5 & \pm 0.00004 & $\pm 0.02 \%$ & \pm 0.005 & \pm 0.025 & \pm 0.035 & \pm 0.020 & \pm 0.4 \\
\hline & 9 & 3 & 0.5 & & & & & comp. & & \\
\hline \multirow[t]{2}{*}{5} & 10 & 3 & 0.5 & \pm 0.00004 & $\pm 0.02 \%$ & \pm 0.005 & \pm 0.025 & \pm 0.035 & \pm 0.020 & \pm 0.4 \\
\hline & 11 & 3 & 0.5 & & & & & & & \\
\hline \multirow[t]{2}{*}{6} & 12 & 3 & Asp & \pm 0.00004 & $\pm 0.02 \%$ & \pm 0.010 & \pm 0.050 & \pm 0.035 & \pm 0.020 & \pm 0.4 \\
\hline & 13 & 3 & 0.5 & & & & & & & \\
\hline \multirow[t]{2}{*}{7} & 14 & 3 & 1 & \pm 0.00004 & $\pm 0.02 \%$ & \pm 0.005 & \pm 0.025 & \pm 0.035 & \pm 0.020 & \pm 0.4 \\
\hline & 15 & 3 & 1 & & & & & comp. & & \\
\hline
\end{tabular}

ETD - maximum edge thickness minus minimum edge thickness (TIR)

Fringes power and irregularity - difference from test plate @ 632 nm

$\mathrm{dN}$ - refractive index difference, $\mathrm{dV}$ - Abbe number change

Surface 7 Asphere Tolerance: less than 0.07 microns peak to valley over any $14 \mathrm{~mm}$ diameter subaperture.

Surface 12 Asphere Tolerance: less than 0.05 microns peak to valley over any $17 \mathrm{~mm}$ diameter subaperture. 


\section{RRU As-Built Fabrication + Alignment Results}

\begin{tabular}{|c|c|c|c|c|c|c|c|c|c|}
\hline Lens-Serial\# & Sur. & $\begin{array}{l}\text { Fringes } \\
\text { (power) }\end{array}$ & $\begin{array}{c}\text { Fringes } \\
\text { (irregularity) }\end{array}$ & $\begin{array}{l}d N \\
d^{*}\end{array}$ & $\begin{array}{c}\text { Lens } \\
\text { wedge } \\
\text { (ETD mm) }\end{array}$ & $\begin{array}{l}\text { Lens } \\
\text { thickness } \\
\text { (mm) }\end{array}$ & $\begin{array}{c}\text { Axial } \\
\text { position } \\
\text { (mm) }\end{array}$ & $\begin{array}{c}\text { Radial } \\
\text { decenter } \\
\text { (mm) }\end{array}$ & $\begin{array}{l}\text { Lens tilt } \\
\text { (arc min) }\end{array}$ \\
\hline \multirow[t]{2}{*}{$1-S N 3$} & 1 & 0.52 & 0.281 & Comp. & 0.003 & +0.020 & 0 & 0.0042 & 0.285 \\
\hline & 2 & 0.67 & 0.329 & & & & & & \\
\hline \multirow[t]{2}{*}{$2-S N 10$} & 3 & 0.07 & 0.221 & Comp. & 0.003 & +0.009 & -0.013 & 0.0032 & 0.252 \\
\hline & 4 & 0.62 & 0.156 & & & & & & \\
\hline \multirow[t]{2}{*}{$3-S N 1$} & 6 & 1.95 & 0.156 & Comp. & 0.005 & -0.010 & +0.018 & 0.0093 & 0.323 \\
\hline & 7 & 1.50 & Asp** & & & & & & \\
\hline \multirow[t]{2}{*}{ 4-SN2 } & 8 & 1.20 & 0.433 & Comp. & 0.003 & +0.023 & -0.013 & 0.0020 & 0.288 \\
\hline & 9 & 1.70 & 0.227 & & & & & & \\
\hline \multirow[t]{2}{*}{ 5-SN1 } & 10 & 0.00 & 0.143 & Comp. & 0.005 & +0.018 & +0.003 & 0.0031 & 0.378 \\
\hline & 11 & 2.00 & 0.250 & & & & & & \\
\hline \multirow[t]{2}{*}{$6-\mathrm{SN} 3$} & 12 & 1.50 & Asp** & Comp. & 0.005 & -0.036 & -0.023 & 0.0013 & 0.052 \\
\hline & 13 & 1.80 & 0.200 & & & & & & \\
\hline \multirow[t]{2}{*}{ 7-SN1 } & 14 & 1.50 & 0.464 & Comp. & 0.000 & +0.013 & 0 & 0.0068 & 0.153 \\
\hline & 15 & 0.07 & 0.069 & & & & & & \\
\hline
\end{tabular}

Fabrication and alignment results good to a fraction of tolerance allocation! Compensation of Lens 1 and barrel-to-barrel spacing were not necessary. Qual/Flight goal is to build to the above tolerances and avoid difficult and time intensive interferometric active alignment. 